\title{
The management of the patient with acute pancreatitis: from evidence to clinical practice
}

\author{
Cristina Cenci, ${ }^{1}$ Tea Restuccia, ${ }^{2}$ Paola Gnerre, ${ }^{3}$ Irene Chiti, ${ }^{4}$ Laura Betti, ${ }^{5}$ Milena Micati, ${ }^{6}$ Claudio Tana ${ }^{7}$ \\ ${ }^{1}$ General Medicine, S. Matteo degli Infermi Hospital, Spoleto USL Umbria 2, Spoleto (PG); ${ }^{2}$ Cardio-Metabolic Internal Med- \\ icine, Nuovo Ospedale di Prato, Usl Toscana Centro, Prato; ${ }^{3}$ Internal Medicine, San Paolo Hospital, Savona; ${ }^{4}$ Internal Medi- \\ cine, USL Toscana Centro, Pescia (PT); ${ }^{5}$ Emergency Department, AUO Careggi, ASL Firenze; ${ }^{6}$ Internal Medicine, Hospital \\ of Galatina, ASL Lecce, Galatina (LE); ${ }^{7}$ Internal Medicine, Ospedale Civile di Guastalla, AUSL Reggio Emilia, Italy
}

\begin{abstract}
Acute pancreatitis is an inflammatory disorder of the pancreas characterized by abdominal pain and elevation of pancreatic enzymes in the blood. The pathogenesis is complex and partly unknown and the evolution is often unpredictable. Many efforts have been made to define this disease and its complications and to classify different grades of severity in order to formulate prognostic scores that could guide the physician in choosing the optimal therapeutic setting and procedures. The management of the patient with pancreatitis is not always optimal and differs among internist, gastroenterologist or surgeon. We think that a patient with clinical suspicion of acute pancreatitis is admitted to medical or surgical department depending on the availability of beds and not according to evidence-based medicine. The aim of this monograph is to identify the optimal management of patients with acute pancreatitis admitted to hospital.
\end{abstract}

\section{Introduction}

Acute pancreatitis (AP) is an inflammatory disorder of the pancreas characterized by abdominal pain and elevation of pancreatic enzymes in the blood. The management of the patient with pancreatitis is not always optimal and differs among internist, gastroenterologist or surgeon. ${ }^{1}$ We think that a patient with clinical suspicion of acute pancreatitis is admitted to medical or surgical department depending on the availability of beds and not according to evidencebased medicine. ${ }^{2}$ The aim of this monograph is to

Correspondence: Cristina Cenci, General Medicine, S. Matteo degli Infermi Hospital, Spoleto USL Umbria 2, Spoleto (PG), Italy.

E-mail: cryscenci73@yahoo.it

Key words: Pancreatitis; guidelines; management.

Conflict of interest: the authors declare no potential conflict of interest.

Received for publication: 9 June 2016.

Revision received: 4 November 2016.

Accepted for publication: 17 November 2016.

This work is licensed under a Creative Commons Attribution NonCommercial 4.0 License (CC BY-NC 4.0).

(C) Copyright C. Cenci et al., 2017

Licensee PAGEPress, Italy

Italian Journal of Medicine 2017; 11:220-245

doi:10.4081/itjm.2017.748 identify the optimal management of patients with acute pancreatitis admitted to hospital.

\section{Epidemiology}

$\mathrm{AP}$ is one of the most common gastroenterological diseases requiring hospital admission. Its incidence varies between 4.9 and 73.4 cases per 100,000 worldwide with an increasing trend. ${ }^{3-7}$ In the US, it has been reported from 13 to 45 cases per 100,000 persons, ${ }^{8,9}$ causing 270,000 hospital admissions annually with an estimated 2.6 billion dollars per year inpatient costs. Incidence and etiology may vary in different countries in relation to different data recording methods, diagnostic criteria and facilities and local risk factors. ${ }^{10}$ About $20-30 \%$ of patients with AP have recurrence and about $10 \%$ develop chronic pancreatitis. Mortality is approximately $1 \%{ }^{9}$

AP equally affects men and women and risk increases with aging. Risk factors include alcohol, smoking, obesity and diabetes. A recent meta-analysis showed that obesity (expressed as body mass index superior to 30) is associated with increased risk of AP and severity of disease. ${ }^{11}$ Diabetes increases AP risk of 1.5-3 folds. ${ }^{9}$

\section{Pathogenesis}

Pathogenesis of this disease is not fully understood. Various experimental models have been used during decades. The most common are: the use of secretagogues (the most frequently used is cerulein, a cholecystokinin analogue. In this model pancreatitis is usually mild); the ligation of common bile duct (re- 
sembling the common channel hypothesis proposed for gallstone pancreatitis in humans, results in bile reflow into the pancreatic duct, which causes inflammation); a choline deficient/ethionine-supplemented diet (that induces hemorrhagic pancreatitis); the infusion of bile salt (sodium taurocholate is directly infused in the pancreatic duct, rapidly causing hemorrhagic necrosis, likely due to a detergent effect); the injection of arginine (intraperitoneal injection of L-arginine induces necrotizing pancreatitis, with a not yet clearly understood mechanism). None of these models is strictly comparable to human condition.

None of these models is strictly comparable to human condition. Moreover, although several conditions are recognized as risk factors for AP, only a small percentage of patients eventually develop the disease (for example only 3 to 7 percent of patients with gallstones and 10 percent of alcoholics), suggesting the interplay of more conditions, triggers and mechanisms.

However, early phase is characterized by inappropriate activation of proteolytic enzymes. In the second phase, intrapancreatic inflammation takes place and in the third phase extrapancreatic manifestations prevail. This scheme is based on the classical theory of Chiari, elaborated more than 100 years ago. The generation of large amounts of trypsin from trypsinogen, triggers an inciting event leading to autodigestive injury of the gland.

In the last years intracellular activation of inflammatory signaling has been studied. Nuclear factor kappa B seems to play a prominent role in the inflammatory cascade of AP. Its activation occurs early and independently from trypsinogen activation. ${ }^{12}$

Other cytokines involved in inflammatory response are tumor necrosis factor (TNF) alpha, interleukin (IL)-1, IL-6, platelet activating factor, intercellular adhesion molecule-1, IL-8, growth-related oncogene alpha/cytokine-induced neutrophil chemoattractant, monocyte chemoattractant protein1 , and substance P.

\section{Pathophysiology}

The revised classification of Atlanta criteria ${ }^{4}$ identifies two phases of the disease: early and late. The first (within 1 week) is characterized by the systemic inflammatory response and/or organ failure and it is classified as mild, moderate or severe. The second is characterized by local and systemic complications.

In mild $A P$, the most common, there is no organ failure, local or systemic complications and resolution usually occurs in the first week.

Moderately severe $A P$ is defined by the presence of transient organ failure, local complications or exacerbation of co-morbidities.

Severe $A P$ is defined by persistent organ failure. The modified score of Marshall, based on three organ systems dysfunctions, considers organ failure in case of a score greater than 1 . This score has the advantage of being simple, universally applicable and allows a stratification of patients easy and objective but has not been validated.

Local complications: acute peripancreatic fluid collections, pseudo cyst, acute necrotic collections and walled-off necrosis, thrombosis splenic and portal, and colonic necrosis syndrome and gastric outlet dysfunction.

Systemic complications are considered exacerbations of pre-existing conditions such as chronic obstructive pulmonary disease or coronary artery disease.

\section{Etiology}

Causes of acute pancreatitis are summarized in Figure 1.

Gallstones are the most common cause of AP in many studies. ${ }^{9}$ A review of 18 studies from European countries confirmed these data with the exception of Denmark and Sweden, where alcohol was the most common cause. ${ }^{7}$ In Italy a study on 1005 patients confirmed biliary etiology in $60 \%$ of patients. ${ }^{13}$ Risk of gallstones pancreatitis increases with age and is higher in women. ${ }^{9}$ Other causes of ampullary obstruction associated with pancreatitis include biliary ascariasis, periampullary diverticula, pancreatic and periampullary tumors, intraductal papillary mucinous neoplasms.

Considering different countries, the role of alcohol varies because of different life style. In the Unites States it accounts for the $30 \%$ of cases. ${ }^{14}$ In Europe it is the second cause of pancreatitis in most countries, with the exception of Sweden and Denmark. Differences are also seen in different regions of the same country. ${ }^{7}$ In the Italian multicenter survey on AP alcohol was responsible for $8.5 \%$ of AP (cases). ${ }^{13}$ Studies on different quantity of alcohol intake gave different results. Binge drinking does not appear to increase the risk of AP in general population. The risk seems indeed to be higher in subjects with a previous history of heavy drinking. ${ }^{9}$

Alcohol is undoubtedly associated with AP but only $5 \%$ of drinkers develop pancreatitis, suggesting that it is not sufficient to determine the disease. Additional insult could be considered: smoking, high fat diet, obesity, genetics and infectious agents. The mechanism by which alcohol induces AP is not well understood. It has been proposed that it can cause biochemical and molecular changes in acinar cells that sensitize the pancreas to injury.

Metabolic abnormalities predisposing to AP include hypercalcemia and hypertriglyceridemia (usually more than $1000 \mathrm{mg} / \mathrm{dL}$ ) that account for 1 to $4 \%$ of cases of acute pancreatitis. Type V hyperlipidemia, as well as types I and IV are prominent causes of acute pancreatitis. Secondary hyperlipidemia is caused by alcohol in- 
take, pregnancy, estrogen therapy, and diabetes. Regarding hypercalcemia, it is, independently from its cause, a rare cause of pancreatitis. Calcium deposition in pancreatic duct and calcium activation of fibrinogen have been proposed as possible mechanisms.

Pancreatitis is a common complication of endoscopic retrograde cholangiopancreatography (ERCP). A systematic survey of studies from 1987 to 2003 found a $3.5 \%$ of post ERCP pancreatitis with $0.4 \%$ of patients experiencing severe AP and $0.11 \%$ death. ${ }^{15}$ Various mechanisms have been proposed: mechanical injury following instrumentation of papilla or thermal injury or chemical insult following injection of contrast medium, intraluminal activation of proteolytic enzymes, infections. ${ }^{16}$

Drugs from different classes are a recognized cause of AP. Drug induced acute pancreatitis (DIAP) represents $0.1 \%-2 \%$ of overall cases. In most cases DIAP seems to be due to an idiosyncratic effect, that is to say an unpredictable abnormal interaction between the drug and the organism. Drugs may act inducing sphincter of Oddi dysfunction, like opioids or causing a pseudolithiasis like ceftriaxone, or increasing the risk of gallstones, like HMG-CoA-reductase-inhibitors. They may also generate toxic metabolites (like nucleoside reverse tran- scriptase inhibitors) or induce hypetriglyceridemia (like estrogens), or give an immuno-mediate reaction (like sulfonamides). ${ }^{17}$ Drugs inducing pancreatitis are classified in four classes: Ia (drugs with at least one case report, evidence of a positive re-challenge, and exclusion of other causes of AP, such as codeine, cytarabine, dapsone, enalapril, furosemide, isoniazid, mesalamine, metronidazole, pentamidine, pravastatin, simvastatin, sulfamethoxazole, sulindac, tetracycline, valproic acid), $\mathrm{Ib}$ (the same but without exclusion of other causes of AP such as amiodarone, azathioprine, dexamethasone, lamivudine, losartan, 6-MP, premarin, trimethoprim-sulfamethoxazole), II (at least four case reports with a consistent latency period for at least $75 \%$ of the cases, for example: acetaminophen, clozapine, erythromycin, estrogen, propofol, tamoxifen), III (at least two case reports but without re-challenge data or a consistent latency period, like alendronate, carbamazepine, ceftriaxone, clarithromycin, cyclosporin, hydrochlorothiazide, ribavirin, metformin, minocycline, naproxen, prednisone, prednisolone), and IV (one case report without re-challenge data, for example ampicillin, cisplatin, colchicine, cyclophosphamide, diclofenac, doxorubicin, interleukin-2, octreotide, propoxyphene, rifampin, risperidone, sertraline, tacrolimus, vincristine).

Biliary gallstones, biliary sludge

Alcohol
$\quad$ Mechanical obstructions to flow of pancreatic juice
$\checkmark \quad$ Ampullary: tumors, stricture or dysfunction of SOD
Metabolic: hypertriglyceridemia hypercalcemia
Drugs
Trauma: Blunt and penetrating, instrumentation (ERCP, pancreatic biopsy)
Ischemia: $\quad$ Hypotension, arteritis, embolic
Hypothermia
Infections: Virus, bacteria and mycobacteria and parasites
Toxins and venoms (spider)
Autoimmune: may be associate with autoimmune diseases (sicca syndrome, PBC, autoimmune hepatitis,
celiac disease)
Genetic (familial, sporadic)
Anatomic variants: Pancreas divisum, choledochal cyst, duodenal diverticula
Idiopathic

Figure 1. Causes of acute pancreatitis. SOD, sphincter of Oddi dysfunction; ERCP, endoscopic retrograde cholangiopancreatography; PBC, primary biliary cirrhosis. 
Acute pancreatitis has also been reported during therapies with interferon (both in the standard and pegylated form) in patients with chronic hepatitis B and $\mathrm{C}$ (in these cases in association with ribavirin), may be due to immune modulation effects of the drug. ${ }^{18-20}$

Also therapies with tyrosine kinase receptor inhibitors (sorafenib and axitinib) could be complicated by acute pancreatitis (with a very low incidence, despite the common detection of hyperamylasemia and hyperlipasemia)..$^{21,22}$

Autoimmune pancreatitis is a rare cause of the disease and may present as acute or chronic. Two types with different histopathologic patterns have been identified. Type 1 is characterized by high levels of IgG4 and is associated to other extrapancreatic manifestations of IgG4 related disease; in type 2, that may be associated to inflammatory bowel disease, levels of IgG4 re normal. ${ }^{23}$

Other causes of AP may be: trauma (both blunt and penetrating, and also instrumentation, e.g. ERCP), ischemia, arteritis (for example in lupus). Infectious agents can also cause AP: Mumps, Coxackie, cytomegalovirus, Salmonella typhi, Leptospira, Legionella, Aspergillus, Toxoplasma, Mycoplasma, Cryptosporidium, Mycobacterium tuberculosis and other mycobacteria. Among viral infections we have to remember HIV (in which pancreas can be affected by the virus itself or be damaged by drugs) $)^{14,4}$ and hepatitis viruses. In fact, AP has been reported during hepatitis due to hepatits A, B, C, E virus. ${ }^{24}$ Both fulminant and, rarely, non-fulminant hepatitis may cause AP. ${ }^{25}$ Pancreatic damage may be due to direct inflammation and destruction of pancreatic acinar cell by the hepatitis virus, ${ }^{26}$ or related to immune response against virus. ${ }^{27}$

We also have to remember toxins, such as organophosphate pesticides, venoms of same arachnids and reptiles.

Structural abnormalities may predispose to AP, such as pancreas divisum (but only few patients develop AP in spite of frequency of this condition that interest $7-8 \%$ of white people), choledochal cyst. In this category, the most harmful are malignancies since tumors may cause mechanical obstruction at ampullary or ductal level and AP episodes may precede overt appearance of the neoplasia. ${ }^{14}$ Sphincter of Oddi dysfunction is due to stenosis or spasm of the sphincter.

Finally, we have to remember idiopathic pancreatitis recently defined as the third cause of pancreatitis. Further insights in etiology and pathophysiology should allow the decrease of unexplained cases.

\section{Diagnosis}

The American College of Gastroenterology guidelines $^{3}$ state that two of these three findings should be present for the diagnosis of acute pancreatitis: i) abdominal pain consistent with the disease; ii) serum amylase and/or lipase greater than three times the upper limit of normal; and/or iii) characteristic findings from abdominal imaging (strong recommendation, moderate quality of evidence).

The new classification divides AP in three degrees of severity: mild, moderately severe and severe. ${ }^{4}$

The mild form is characterized by the absence of organ failure and local or systemic complications. It is not required to perform imaging methods and mortality is almost absent. Discharge within the first week. The moderately severe form is characterized by the presence of organ failure or transient local or systemic complications in the absence of renal organ persistent. It may resolve without intervention or may require prolonged specialized care. Its mortality rate is far lower than that of the severe form. The severe form is characterized by failure organ persistent $(>48 \mathrm{~h})$. When systemic inflammatory response syndrome (SIRS) is present, there is an increased risk of severe pancreatitis, and in the presence of persistent organ failure since the early days mortality can be very high and is reported up to $36-50 \%$.

\section{Clinical presentation}

Most patients with acute pancreatitis have acute onset of persistent, severe epigastric abdominal pain. ${ }^{28}$ In some patients, the pain may be in the right upper quadrant or, rarely, confined to the left side. In patients with gallstone pancreatitis, the pain is well localized and the onset of pain is rapid, reaching maximum intensity in 10 to $20 \mathrm{~min}$. In contrast, in patients with pancreatitis due to hereditary or metabolic causes or alcohol, the onset of pain may be less abrupt and the pain may be poorly localized. In approximately 50 percent of patients, the pain radiates to the back..$^{29}$ The pain persists for several hours to days and may be partially relieved by sitting up or bending forward. Approximately 90 percent of patients have associated nausea and vomiting which may persist for several hours. ${ }^{30}$

Patients with severe acute pancreatitis may also have dyspnea due to diaphragmatic inflammation secondary to pancreatitis, pleural effusions, or adult respiratory distress syndrome. Approximately 5 to 10 percent of patients with acute severe pancreatitis may have painless disease and have unexplained hypotension (e.g., postoperative and critically ill patients, patients on dialysis, organophosphate poisoning, and Legionnaire's disease)..$^{31-35}$

Finally, to evaluate the evolution of pancreatitis it is important to determine the exact interval between symptom onset and hospitalization and in case of transfer center specializing in the interval between the first admission and transfer. ${ }^{4}$

\section{Physical findings}

Physical findings vary depending on the severity of acute pancreatitis. In patients with mild acute pan- 
creatitis, the epigastrium may be minimally tender to palpation. In contrast, in patients with severe pancreatitis, there may be significant tenderness to palpation in the epigastrium or more diffusely over the abdomen. Patients may have scleral icterus due to obstructive jaundice caused by choledocholithiasis or edema of the head of the pancreas.

Patients with severe pancreatitis may have fever, tachypnea, hypoxemia, and hypotension. In $3 \%$ of patients with acute pancreatitis, ecchymosis discoloration may be observed in the periumbilical region (Cullen's sign) or along the flank (Grey Turner sign). ${ }^{35}$

These findings, although nonspecific, suggest the presence of retroperitoneal bleeding in the setting of pancreatic necrosis. ${ }^{36}$ Patient may also have findings suggestive of the underlying etiology. As examples, hepatomegaly may be present in patients with alcoholic pancreatitis, xanthomas in hyperlipidemic pancreatitis, and parotid swelling in patients with mumps.

\section{Laboratory findings}

The two main enzymes that aid diagnosis of acute pancreatitis are amylase and lipase. Serum amylase in AP patients generally rises within a few hours after the onset of symptoms and normalizes within 3-7 days. On admission, it can remain in the normal range in a fifth of patients. ${ }^{37,38}$ Compared with lipase, serum amylase returns more quickly to values below the upper limit of normal. Due to the low sensitivity and specificity serum amylase alone cannot be used reliably for the diagnosis of AP.

Lipase rises within 4-8 $\mathrm{h}$ and stays elevated for 814 days. Lipase levels of greater than five times the upper limit of normal have $100 \%$ specificity for the diagnosis of AP. Lipase is now the laboratory measurement of choice for the diagnosis of AP. Serum lipase or amylase levels at admission do not predict severity of disease. Other enzymes can be elevated in AP. Trypsinogen activation peptide (TAP), a five amino-acid peptide that is cleaved from trypsinogen to produce active trypsin, is elevated in acute pancreatitis. Since activation of trypsin is likely an early event in the pathogenesis of acute pancreatitis, TAP may be useful in the detection of early acute pancreatitis and as a predictor of the severity of acute pancreatitis. ${ }^{39-42}$ Urinary and serum trypsinogen-2 levels are newer tests and are elevated in early acute pancreatitis. They are not readily available and additional studies are needed to determine their role in the diagnosis of acute pancreatitis. ${ }^{43-46}$

Acute pancreatitis is also associated with elevations in C-reactive protein (CRP), IL-6, IL-8, IL-10, TNF, and PMN elastase..$^{46}$ A CRP level above $150 \mathrm{mg} / \mathrm{dL}$ within $48 \mathrm{~h}$ is associated with severe pancreatitis.

Additional laboratory tests that are useful for the diagnosis of AP are liver function tests and hematocrit.
In patients with no history of alcohol consumption, the presence of alanine aminotransferase (ALT) elevation three times the upper limit of normal has a $95 \%$ positive predictive value for acute gallstone pancreatitis. ${ }^{47}$ Normal liver function tests do not exclude the diagnosis of biliary pancreatitis, as this can occur in up to $20 \%$ of patients. ${ }^{48}$ Hemoconcentration (defined as hematocrit greater than $44 \%$ ) and failure of hematocrit to decrease at $24 \mathrm{~h}$ are important predictors of severe pancreatitis. Metabolic abnormalities including elevated blood urea nitrogen (BUN), hypocalcemia, hyperglycemia, and hypoglycemia may also occur. Clinical conditions that may be associated with increased levels of amylase in the absence of AP are: macroamylasemia (a syndrome characterized by the formation of large molecular complexes between amylase and abnormal immunoglobulins); decreased glomerular filtration rate; diseases of the salivary glands; extrapancreatic abdominal diseases associated with inflammation (acute appendicitis, cholecystitis, intestinal obstruction or ischemia, peptic ulcer, and gynecological diseases).

\section{Role of ultrasound in acute pancreatitis}

Ultrasound (US) represents the first-line imaging technique in the assessment of pancreatic disease. It has several advantages as compared to contrast-enhanced computed tomography (CT) and magnetic resonance imaging (MRI), such as low cost, real time evaluation and no radiation exposure. ${ }^{49}$ US can be performed easily at patient's bedside and demonstrates good values of sensitivity and specificity in the assessment of AP (67 and $100 \%$, respectively).$^{50}$ Furthermore, US is useful in the follow-up of the patients, revealing local complications such as abscesses, necrosis, peripancreatic fluid, pseudocysts, abscesses and vascular disease (venous thrombosis and pseudoaneurysms).

The pancreas, however, is sometimes difficult to explore. Patients are often obese, and meteorism can limit its evaluation, for example in the case of local distension of intestinal loops by pancreatic inflammation (sentinel loop). In these cases, the visualization can be improved by water filling and changing in position (from supine to upright). With these measures, the pancreas can be fully displayed in a large percentage of patients. ${ }^{51}$

\section{Conventional ultrasound: B-mode and color Doppler findings}

The inflammation can involve diffusely the pancreatic gland or only some portions such as the head and body. On conventional US, the gland appears enlarged but this feature can be not so evident in the case of mild, edematous pancreatitis. An anteroposterior size of the body greater than $24 \mathrm{~mm}$ is traditionally suggestive of increased gland. ${ }^{52}$ 
Some authors, however, believe that the increase in size of the pancreas in the course of acute pancreatitis should be considered relative to the gland size in normal conditions, as the latter may vary from subject to subject and should not be strictly correlated to an absolute value.

A more pronounced edema can manifest as mass effect on the adjacent organs (gastric antrum, veins regional site, bile duct), suggesting the increase in pancreas size, avoiding further measurement of the gland in the correspondence of the body. Margins can appear unchanged or irregular, blurred or polycyclic in the case of severe disease. ${ }^{53}$

\section{Echogenicity}

The gland appearance on B-mode US varies in relation to the clinical stage and severity of pancreatitis. In mild disease, the echogenicity is similar to that observed in normal conditions. A more pronounced inflammatory damage of the gland appears instead diffusely hypoechoic in the edematous form or occur with appreciable inhomogeneity with the necrotic-hemorrhagic involvement. The necrotic areas, in particular, can appear hypoechoic as compared to the healthy parenchyma, and their recognition is important because often enters in the differential diagnosis with neoplastic disorders such as pancreatic adenocarcinoma. A correct diagnosis is based not only on the US features but should be integrated also on clinical and laboratory findings. ${ }^{52,53}$ The appearance of hemorrhagic areas varies on US depending on the state of red blood cells aggregation and the phase in which it is detected. In the early stages, acute phase the hemorrhagic areas are hyperechoic; in the late stages, the echogenicity decreases following the clot rupture of the clot. ${ }^{53}$ Furthermore, the inflammation of the retroperitoneal tissue can be seen sometimes as a hypoechoic, perivascular halo around the portal and splenic veins. ${ }^{54}$

\section{Color Doppler ultrasound findings}

Color Doppler US (CDUS) is an important step in the assessment of pancreas and its vascular structures, in particular the portal vein, splenic, mesenteric arterial and venous vessels, aorta and inferior vena cava.

While peripancreatic vessels are well assessed on CDUS, only few intraparenchymal vessels can be fully appreciated in normal conditions.

However, the increasing diffusion of high sensitive US devices can allow a greater visualization of small, peri and intrapancreatic vessels, that is particularly important in the evaluation of vascular complications of acute pancreatitis such as venous thrombosis and pseudoaneurysms. ${ }^{55}$

Portal vein thrombosis can manifest on CDUS as partial or complete absence of flow within intraluminal echogenic areas. CDUS can also be reliable to evaluate suspected pseudoaneurysms arising from mesenteric or splenic arteries, secondary to tunica media disruption by pancreatic inflammation; pseudoaneurysms appear as small anechoic lesions on B-mode but their vascular origin can be revealed by CDUS that show turbulent flow or typical mosaic pattern on color Doppler and arterial flow on spectral analysis..$^{53}$

\section{Evaluation of common bile and pancreatic ducts}

US evaluation of acute pancreatitis is not complete without the study of biliary and pancreatic ducts. Cholelithiasis is most frequently associated with acute pancreatitis in developed countries. Gallstones appear as hyperechoic, intraluminal formations showing posterior shadow cone, fully movable on changing the patient's position, thus leading to the so-called rolling stone sign in case of movement along the rear wall. Duct stones in the common bile duct (CBD) have similar US characteristics; however, the mobility with the change of the position of the patient can be less easily appreciated. The biliary sludge can manifest as multiple echoes slowly floating with changes of decubitus and usually distributes along the rear wall of the gallbladder or within the CBD lumen. It usually does not generate posterior shadow cone, differently from traditional stones. ${ }^{56}$

CBD stones can result often in the dilatation of intra or extrahepatic bile ducts, the former evident with the classic pruned-tree appearance, due to the presence of enlarged and convergent intrahepatic common bile ducts. CBD dilatation can be easily recognized as exceeding in general $8 \mathrm{~mm}$ of diameter. Rarely, CBD stones can be associated with dilatation of duct of Wirsung; in these cases, the dilatation derives usually from mass effect secondary to the focal edema in the head of the pancreas. Under normal conditions the size does not exceed 2-3 mm; a diameter exceeding $3 \mathrm{~mm}$ must raise the suspicion of obstructive and/or inflammatory etiology. ${ }^{53,55}$

Other US signs that can be found in association with AP are cholecystitis and cholangitis. ${ }^{53}$

\section{Locoregional and extra-glandular complications}

Complications of acute pancreatitis are classified into locoregional, if they grow in close proximity of the pancreatic gland affected by inflammation, and extra-glandular, if are localized outside of the pancreatic parenchyma. The presence of inflammatory exudate may be associated with the formation of fluid collections that can localize in or around the pancreatic gland, sometimes under the capsular layer or outside the gland, inside adjacent organs (liver, spleen and kidneys). The sonographic features of such collections consist of areas with not well-defined borders with hypo-anechoic echostructure, which sometimes include reinforcement of the wall due mostly to the 
presence of fat edematous and infiltrated. The feature of different echostructural homogeneity is usually due to the presence of echogenic debris, septa and/or hemorrhagic fluid. ${ }^{53}$

The fluid collections located under diaphragm muscle can propagate in the pericardial, pleural or mediastinal cavities; in about the half of cases they regress spontaneously; Otherwise, if not reabsorbed, they may be organized in structures better defined as pseudocysts. They appear, when studied through Bmode examination, as not homogeneous areas, with variously echogenic mobile iso-anechoic content, covered with thickened wall with reinforced rear and mostly regular margins. The pancreatic bed on which pseudocysts are located is irregular with echogenic fragments; the latter are the result of necrotic and autolytic processes. ${ }^{53}$ Usually there is no vascular signal at CPD examination.

In this context, ultrasound contrast-enhanced ultrasound (CEUS) can add useful information for the differential diagnosis between pseudocyst and cystadenoma. The pseudocyst usually appears as non-enhanced and also the intraluminal echogenic component, if present, does not tend to take ultrasound contrast agent. Pseudocysts newly formed can have a wall hyperenhanced, unlike the old ones that have, instead, hypoenhanced walls. ${ }^{57}$

Rarely pancreatic pseudocysts may be confused with bilomas, rare collections of abnormal bile in the intra- or extra-hepatic due to a spontaneous or iatrogenic interruption of bilious system. The sonographic appearance may be almost comparable to that of pseudocyst. The suspected diagnosis will be placed according to an history of previous trauma or surgery of the biliary tree, and confirmed by the imaging of second level (MR cholangiopancreatography), which will highlight the close relationship of continuity of the lesion with the biliary tree..$^{58}$

Another locoregional complication is represented by abscess or by superinfection of a fluid collection or by liquefaction of a necrotic area. It may constitute a serious complication that can lead to abrupt clinical deterioration and that needs, therefore, timely assessment of advanced cares. An abscess should be suspected in case of signs of infection (e.g., neutrophilic leukocytosis and fever) and the occurrence of abdominal pain; the sonographic appearance consists of an increase in the volume of the collection with onset within echogenic material sometimes sloping or fluctuating.

Another serious complication is represented by infected pancreatic necrosis. It has no sonographic features, which make it distinguishable from the other fluid collections, and usually it occurs with the appearance of fine small echoes within the liquid, variously mobile and floating. ${ }^{53}$

\section{Role of contrast-enhanced ultrasound}

In the last decade, conventional B-mode US has been improved by CEUS that has improved the diagnostic abilities of ultrasound thanks to a great definition of vessels and microvessels of the examined districts.

US contrast agents (UCAs) are exogenous substances that can be administered intravenously to enhance the ultrasound signal. All agents contain gas-filled microbubbles with a diameter of $2-6 \mu \mathrm{m}$, surrounded by a shell composed of varying lipids or polymers. The gas and the shell influence the half-lives of the microbubbles and their response to insonation.

When hit by US, the microbubble sends back to the transducer a wave that has a frequency equal to that of insonation and a series of harmonics of the fundamental frequency. Among these, the second harmonic has a frequency double than the fundamental. The harmonic response is high as the acoustic pressure of the incident wave, but causes a greater destruction of microbubbles. If the microbubbles have elastic membrane, it is possible to apply a lower acoustic pressure to generate a good harmonic response. Sonovue has a better harmonic response with a frequency of 3 to $3.5 \mathrm{Mhz}$; therefore, the second harmonic is 6-7 MHz. The structures produce both the fundamental frequency and harmonics of fundamental frequency (tissue harmonic imaging) generated by the distortion of the wave by crossing tissue; these harmonics are weaker than those produced by the microbubbles and employing dedicated platforms is possible to distinguish them from harmonics generated by the microbubbles. Harmonic tissue can cause artifacts when operators use higher-pressure acoustic signal.

Second generation contrast agents, such as BR-1 and Sonovue are those mainly used in Europe. Sonovue is composed of sulfur hexafluoride with a phospholipid shell, which provides stability and resistance. Sonovue generates non-linear harmonic frequencies, since at low acoustic power of insonation the degree of microbubbles expansion is greater than its destruction.

These new UCAs oscillate without destruction at low mechanical index, producing harmonic frequencies that are multiples of the transmitted frequency (non-linear fundamental echoes), allowing real-time imaging of microbubble signals. The introduction of a dedicated contrast agent software in the ultrasound equipment enables the visualization of the microbubble signals without the fundamental grey-scale echoes.

Since its infusion, contrast agent takes about 15$30 \mathrm{~s}$ to reach the structures of interest.

Then, gas is eliminated from the lung while the components of the membrane from liver and kidney.

Second-generation contrast agents are not indicated in case of: recent acute coronary syndrome, unstable angina, recent acute heart attack, recent coronary artery 
intervention, acute or class III or IV chronic heart failure or severe arrhythmias. No interaction with other drugs has been reported and only mild and transient adverse reactions have been reported.

CEUS examination should be performed after a conventional B-mode study. The probe used is a multifrequency curved array transducer (3-4 MHz) and an intravenous bolus of $2.4 \mathrm{~mL}$ of contrast agent is administered.

In the study of pancreatic disease, CEUS is not recommended for the detection of pancreatic lesions but it is useful to define more precisely lesions already found through traditional ultrasound examination. ${ }^{59}$

Different patterns of enhancement are related to different pathological conditions, depending on characteristics of the lesions (focal or widespread, several or single, solid or liquid, neoplastic or pseudo-neoplastic). The enhancement pattern of focal pancreatic lesions should be compared with the adjacent pancreatic texture. Therefore, the examination should include the mass under investigation and a portion of surrounding pancreatic parenchyma. In order to understand the meaning of different ways of contrast distribution, it is important to specify that pancreas enhancement is far different from liver enhancement: blood contribution to the pancreas is completely arterial, and CEUS shows an early and brief enhancement of pancreatic gland. Arterial phase is very early (10 to $30 \mathrm{~s}$ with a peak of enhancement to $15-20 \mathrm{~s}$ ), and is followed by a transient venous phase (30 to approximately $120 \mathrm{~s}$ ) during which spleno-mesenteric-portal venous axis is enhanced. The late phase (about $120 \mathrm{~s}$ after injection) is defined by enhancement of the hepatic veins. These characteristics make the study of the pancreas particularly difficult.

As contrast agents have a pure intravascular distribution, CEUS of the pancreas is suitable to discern between solid and cystic lesions, to describe focal masses, and to provide a clear differentiation between surrounding tissue, fibrosis and necrosis.

CEUS improves the diagnosis of the inflammatory pathology of the pancreas and is particularly useful in the staging of the severity of the acute pancreatitis, in the detection of area of necrosis and in the recognition of its complications. ${ }^{60}$

Edematous acute pancreatitis appears at a conventional B-mode examination, as widely described before, with a dimensional increase of the gland and a diffusely hypoechoic texture. The injection of contrast agent will show a diffused and homogeneous wash-in of the gland, with different degrees of enhancement, resulting in an increased echogenicity during the dynamic phases.

Autoimmune pancreatitis is a pathological condition characterized by inflammation around the ducts sustained by lymphocytes migration, which leads to fibrosis. The features showed at B-mode study are similar to focal acute pancreatitis: the gland appears enlarged, hypoechoic and pancreatic duct results expanded. CEUS shows different aspects: some authors have reported enhancements similar to that of the normal pancreatic parenchyma, others have described a mild or high wash-in followed by a slow wash-out of contrast agent. ${ }^{61}$

Focal acute pancreatitis appears at conventional Bmode examination as an expanded area of the pancreas, homogeneously hypoechoic. Sometimes, such images set serious problems of differential diagnosis with pancreatic tumor masses. The principal indication of the CEUS consists in the characterization of pancreatic lesions found at traditional ultrasound performed in a context of an acute pancreatitis. Focal acute pancreatitis is underlined by CEUS as a zone of increased impregnation of contrast agent. Necrotic area appears as an anechoic image on B-mode study without enhancement after contrast injection. ${ }^{62}$

Although the gold standard in the diagnosis of the necrotic areas in severe acute pancreatitis is represented by CT, CEUS constitutes currently the best technique in the follow-up of these patients after CT staging, because it reduces the exposure to radiations. ${ }^{63}$

Moreover, CEUS is useful in the recognition and in the characterization of pancreatic pseudocysts. Such lesions appear at B-mode examination as inhomogeneous areas, circumscribed by a not well-defined wall, containing sometimes echoic mobile material inside, and have a hypo-anechoic texture. CEUS will show no enhancement of the wall of pseudocyst in all phases, even if inhomogeneous on US. The sensitivity and specificity of CEUS in characterizing pseudocysts is up to $100 \%$. Therefore, CEUS has a great importance in the differential diagnosis of pseudocysts and cystic tumors of the pancreas, whose walls result instead hyper-enhanced. Contrast agent injection makes easier the diagnosis of these formations, thanks to a more detailed characterization of the vascular component of cystic inner elements.

As reported in The EFSUMB Guidelines and Recommendations on the Clinical Practice of Contrast Enhanced Ultrasound (CEUS): Update 2011 on non-hepatic applications the recommended uses and indications of CEUS in patients with focal pancreatic lesions identified with US are: i) characterization of ductal adenocarcinoma (Recommendation Level: $\mathrm{A} ; 1 \mathrm{~b})$; ii) differential diagnosis between pseudocysts and cystic tumors (Recommendation Level: A;1b); iii) differentiation of vascular (solid) from avascular (liquid/necrotic) components of a lesion (Recommendation Level: A;1b); iv) defining the dimensions and margins of a lesion, including its relationship with adjacent vessels (Recommendation Level: B;2b); v) management of the lesion with a better distinction between solid and cystic lesions, thus providing information for the choice 
of the next imaging modality (i.e., MRI and/or endoscopic US for cystic lesions) (Recommendation Level: $\mathrm{C} ; 5)$; vi) diagnosis of cases that are indeterminate on CT (vascularization of solid pancreatic lesions; differential diagnosis between pseudocysts and pancreatic cystic tumors, especially mucinous cystic tumor) (Recommendation Level: C;5). ${ }^{64}$

A new technique of imaging, CEUS, combines the advantages of endoscopic US with amplification by contrast agents. ${ }^{65}$ After EUS examination CEUS is used to characterize micro-vascularization of the lesions, to differentiate benign from malignant masses, to improve diagnosis, staging of the lesions and to improve therapeutic procedures.

CEUS can be performed with a high or low mechanical index. This technique can be useful in the differential diagnosis of autoimmune pancreatitis. Focal and diffuse autoimmune pancreatitis result hyper-enhanced at CEUS examination. Some Authors have described that a low MI at CEUS shows hyperenhancement in focal and diffuse autoimmune pancreatitis and allows the differential diagnosis with ductal adenocarcinoma (which does not hyper-enhance). ${ }^{66}$

\section{Role of pancreatic endoscopic ultrasound}

EUS represents a new mild invasive technique of imaging that combines the advantages of B-mode ultrasound with those of endoscopy. Thanks to the proximity of EUS transducer to the organs of interest, the images obtained are frequently more accurate and more detailed than the ones obtained by traditional ultrasound. EUS can obtain information about the layers of the intestinal wall as well as adjacent areas such as bilious ducts, pancreatic structures, lymph nodes and blood vessels. These features have encouraged its use in clinical practice. ${ }^{67}$ Numerous studies have recently testified forthe safety, the accuracy and the indications of this exam, whose real limit is the hold dependence from the experience of the operator.

In the acute inflammatory pathology of pancreas, EUS allows: i) to clearly identify the lithiasis etiology of acute pancreatitis thanks to its ability in detecting stones inside biliary structures or pancreatic ducts; ii) to drain pancreatic pseudocysts; iii) to differentiate between pancreatic pseudocysts and bilious or pancreatic tumors; and iv) to obtain a fine needle aspiration of structures of interest.

Even if not performed during acute pancreatitis it can be used further to clarify the causes of inflammatory process or to treat its complications.

\section{Biliary lithiasis}

The lithiasis of main biliary duct prevails in $20 \%$ of patients affected by gallbladder lithiasis and causes the development of severe complications such as acute pancreatitis and acute cholangitis. The diagnosis of lithiasis of common biliary duct is not always easy and clinical tests and conventional imaging are not endowed with elevated sensitivity. EUS offers, on the contrary, high sensitivity and specificity and represents a valid new diagnostic technique and a reliable alternative to ERCP. The suspicious cases for lithiasis of the principal biliary tract, where traditional ultrasound has not been diagnostic, require the execution of colangio-MRI or EUS. B-mode ultrasound has a low sensitivity in the detection of stones (22-55\%); it rather gives indirect information about the presence of lithiasis through the detection of expansion of the biliary system, with a sensitivity from $77 \%$ to the $87 \%$. The sensitivity of colangio-MRI in the diagnosis of lithiasis of principal biliary duct goes from $85 \%$ to $92 \%$ and the specificity from $93 \%$ to $97 \%$, with a meaningful decrement of this value with decreasing sizes of calculi. The reliability of EUS is not conditioned instead from the dimensions of stones: EUS is able to detect biliary deposits even if they are not seen by ERCP, with a sensitivity between $89 \%$ and $94 \%$, and a specificity that goes from $94 \%$ to $95 \%$. The underestimated biliary lithiasis by ERCP suggests that the echoendoscopic study of the extra-hepatic bilious ducts could represent a valid alternative to obtain diagnostic information. EUS has instead largely reduced the cases of idiopathic pancreatitis and it has progressively limited the use of ERCP in those cases, which need therapeutic endoscopy. The study of the biliary and pancreatic ducts by EUS is technically limited in case of: pneumobilia, outcomes of interventions on stomach and masses of cephalic part of the pancreas. Despite these limits, EUS reaches a sensitivity of $97 \%$ and a specificity of $100 \%$ in the diagnosis of lithiasis of common bile duct, showing a higher diagnostic accuracy than conventional US and $\mathrm{CT}^{68,69}$ and a better safety profile than ERCP. ${ }^{70}$

\section{Drainage of pseudocyst}

Pancreatic pseudocysts are intra or extra-pancreatic fluid collections composed of pancreatic secretions and inflammatory debris. Reactive granulation tissue rather than a true epithelial lining wall surrounds the fluid collection, hence the term pseudocyst. Pseudocysts originate from leaks in the pancreatic duct. The etiology may be necrosis secondary to pancreatitis, progressive ductal obstruction, or trauma.

Most of pseudocysts are asymptomatic and do not require treatment. An enlarging pseudocyst may require drainage in order to avoid rupture or hemorrhage transformation. Pseudocysts that complicate acute pancreatitis have a high probability to spontaneously resolve within 4 to 6 weeks and should be observed in this period before considering further treatment. Earlier drainage may be indicated when clinical pancreatitis fails to improve despite an aggressive medical management. 
Pseudocysts complicating chronic pancreatitis usually result from pancreatic duct outflow obstruction from a stone, stricture, or accumulation of protein materials. Such pseudocysts rarely resolve on their own. Drainage is indicated to relieve symptoms associated with a space-occupying mass and neighboring organ compression such as pain, gastric outlet obstruction, and jaundice. Drainage is also indicated when pseudocysts become infected or if there is intracystic bleeding.

The application of EUS to guide pseudocyst puncture through the stomach or duodenal wall has improved the success and safety of endoscopic pseudocyst drainage. Using endoscopic guidance alone, a prominent mucosal bulge should be present to identify the site for pseudocyst puncture. Even though, the interposed tissue may contain vessels. EUS provides a highly detailed view of the pseudocyst and surrounding topographical anatomy. Surface vessels are readily detected with color Doppler. The use of a sectorial echo-endoscopic tool with a wide channel of puncture ensures safety and easy execution. With this technique, the drain of the pseudocyst is performed easily, reducing significantly operating risks. ${ }^{71}$

\section{Biliary and pancreatic tumors}

The tumors of biliary ducts and pancreatic gland are characterized by high malignancy and difficult surgical treatment. These features determine a low survival. An accurate study of such tumors is desirable to select patients who are candidates for surgical intervention. ${ }^{72}$

EUS has changed radically the study of the tumors of biliary and pancreatic structures: this technique has revealed to be superior to $\mathrm{CT}$ in the staging and in detection of invaded lymph nodes. Moreover, EUS allows performing a fine needle aspiration of the lesion and of the satellite lymph nodes.

Unlikely traditional ultrasound, EUS allows an accurate identification and staging of ampullary tumors and of tumors of the principal biliary duct. ${ }^{73,74}$ The sensitivity and the diagnostic accuracy of EUS respectively reach $100 \%$ and $93 \% .{ }^{75-77}$ Morphologically, the ampullary tumor appears as a hypoechoic area in duodenal papilla.

The diagnostic accuracy of EUS in the diagnosis of carcinoma of the common liver duct and of its bifurcation is about $85 \%$ with B-mode US and arises $91 \%$ if micro-probes are used. ${ }^{76,77}$ Cholangiocarcinoma of the principal biliary ducts appears as a small mass projecting inside the common bile duct or as a thickening of its wall. EUS is suitable in the diagnosis of pancreatic tumors whereas a strong clinical suspect exists in the absence of comforting imaging. EUS sensitivity and specificity to detect pancreatic tumors are $90 \%$ and $89 \%$, respectively and accuracy is $81 \%{ }^{78}$ The superiority of EUS in comparison to CT in the diagnosis of pancreatic tumors increases for lesions smaller than $3 \mathrm{~cm}$ of diameter. ${ }^{79}$ Morphologically, these tumors appear as hypoechoic lesions with poor defined margins or as a mass which compresses the principal biliary tract or pancreatic duct.

Focal pancreatic lesions represent the principal indication for EUS-driven biopsy; fine needle aspiration has a sensitivity of $94 \%$ and an accuracy of $92 \%$. In case of cystic pancreatic lesions, this technique allows to distinguish among benign and malignant forms through a cytological analysis.

\section{Complications}

In comparison with standard endoscopy the risks of perforation are slightly greater because of a higher rigidity of the tool. In case of invasive endoscopy, the risks of bleeding and infection are higher. Such complications strongly depend on the experience of the operator. ${ }^{80}$

\section{Assessment and risk stratification}

The correct clinical evaluation of the patient with AP is critical to identify the proportion of patients (15$25 \%$ ) that will evolve towards a severe form of acute pancreatitis. The ability to predict the severity of the AP allows the identification of patients that benefit from an early transfer to intensive care or specific invasive interventions. In literature, concerning the risk of AP, there are several predictive models based on clinical, laboratory values, radiological risk factors, severity scores and serum markers. ${ }^{81}$ Unfortunately, these predictive models (which can be applied to the patient at the time of access to the emergency room or in the first 48 or $72 \mathrm{~h}$ ) have a low specificity. Low specificity associated with low prevalence of severe AP translate into low positive predictive values. ${ }^{82}$ Table $1^{31,83-101}$ shows the clinical predictors, laboratory and radiological findings of acute pancreatitis gravity.

\section{Scoring system}

There are several scoring systems applied to the patient with AP but none of them has proven to be ideal in predicting the severity of AP. ${ }^{102,103}$ These systems are still superior to clinical judgment to determine which patients are candidates for Intensive Care Unit (ICU) admission and who needs a more aggressive treatment. They are also useful for monitoring the evolution of the AP within some hours from hospitalization. Table $2^{4,104-114}$ summarizes the main features of the score system used in clinical practice.

\section{Therapy of acute pancreatitis}

The treatment of acute pancreatitis is divided into three stages: i) treatment of acute phase; ii) treatment of complication; iii) treatment of predisposing factors. 
Table 1. Clinical predictors, laboratory and radiological of acute pancreatitis gravity.

\begin{tabular}{|c|c|}
\hline Clinical & Laboratory \\
\hline Clinical judgment & Hemoconcentration \\
\hline It is the result of the impression of the & The studies that evaluated the hematocrit \\
\hline physician evaluating the patient with & as a predictor of the severity of AP \\
\hline $\mathrm{AP}$ at the emergency room. & have produced variable results. It seems \\
\hline It is based on clinical and laboratory data. & that a normal or low hematocrit at \\
\hline It has sensitivity specificity, positive & admission and during the first 24 hours \\
\hline $\begin{array}{l}\text { predictive value and negative } \\
\text { predictive value respectively of }\end{array}$ & is generally associated with a milder \\
\hline predictive value respectively of & clinical course $^{88-89}$ \\
\hline
\end{tabular}

\section{Radiological}

\section{Chest $X$-ray}

The presence of pleural effusion or pulmonary infiltrates during the first $24 \mathrm{~h}$ may be associated with pancreatic necrosis and organ failure ${ }^{96}$

\author{
Sex \\ It is not considered a predictor of \\ outcome in many studies ${ }^{31}$
}

\section{C-reactive protein}

The value of the CRP is directly proportional to the severity of pancreatitis. It is an inexpensive and ready available test. CRP value $>150 \mathrm{mg} / \mathrm{L}$ after $48 \mathrm{~h}$ of onset of AP are a watershed between mild and severe forms ${ }^{83}$

\section{$B U N$}

In some studies ${ }^{90,91}$ blood urea was found to be laboratory examination more useful in predicting mortality of AP. Values of $\mathrm{BUN} \geq 20 \mathrm{mg} / \mathrm{dL}$ on admission, are associated with an increased risk of mortality as well as any subsequent increases in blood that occurred within $24 \mathrm{~h}$
Abdominal computed tomography with contrast medium A retrospective analysis of the different scoring systems for the severity of acute pancreatitis based on abdominal CT, found that none were statistically superior to the systems of clinical score (APACHE II or BISAP). On the other hand, abdominal CT input can be negative even in the presence of AP serious because it takes time before the pancreatic necrosis develops and becomes detectable with this strument. One study described the usefulness of abdominal CT perfusion imaging in predicting pancreatic necrosis of the early stages of the AP. Of 10 patients with signs of ischemia pancreatic CT perfusion imaging performed at the entrance 9 had a subsequent confirmation of pancreatic necrosis subsequent CT performed within the next 3 weeks ${ }^{97,98}$ Abdominal magnetic resonance and colangiopancreatic magnetic resonance

Magnetic resonance imaging is of comparable diagnostic and prognostic value with computed tomography in the staging of acute pancreatitis. ${ }^{99}$ One study showed that MRI is reliable for staging the severity of the AP and to predict prognosis with less contraindications than CT. ${ }^{100}$ Furthermore MRI can also detect interruptions of the pancreatic duct, which can occur in the early stages of a AP. MRI plays an important role in the diagnosis and staging of acute pancreatitis ${ }^{101}$

\section{Alcohol}

In many studies, alcohol is associated with an increased risk of pancreatic necrosis with the need for invasive treatments ${ }^{84}$

\section{Serum creatinine}

An elevated serum creatinine within the first 48 hours can predict the development of pancreatic necrosis. ${ }^{91,92}$ A normal creatinine has a high negative predictive value for the development of pancreatic necrosis. A normal creatinine in the absence of complications, may obviate the need to perform an abdominal CT

\section{Other serum markers}

Other serum markers have been variously tested in several clinical studies $^{94,95}$ to predict the severity of the AP, including: urinary trypsinogen Obesity (BMI $>30)$ is a factor predictive
of severe AP in many studies. A meta-analysis ${ }^{85}$ with 739 patients estimated: OR 2.9 (95\% CI 1.8 to 4.6$)$ for serious AP; OR 2.3 (95\% CI 1.4 to 3.8) activation peptide, procalcitonin, for systemic complications;

OR 3.8 (95\% CI 2.4 to 6.6$)$ for local amylase, lipase, polymorphonuclear elastase, pancreatic-associated protein, complications; OR, 2.1 (95\% CI 1.0 to 4.8 ) serum glucose, serum calcium, for mortality pro-carboxypeptidase B

carboxypeptidase $\mathrm{B}$ activation peptide, serum trypsinogen-2, phospholipase A-2, serum amyloid protein-A, substance $\mathrm{P}$, antithrombin III, platelet activating factor, interleukins 1,6 , and 8 , tumor necrosis factor-alpha or soluble tumor necrosis factor receptor, many of which are not even available for daily use, and therefore they will not be considered due to their unproven clinical utility

Short time interval between start of the

symptoms and hospitalization

A time interval less than $24 \mathrm{~h}$ is

associated with increased severity

of pancreatitis ${ }^{86}$

Organ failure

The presence of early and persistent

organ failure is widely regarded as a

predictor of severe AP, increased

mortality and prolonged hospital stay ${ }^{87}$

AP, acute pancreatitis; CRP, C-reactive protein; CT, computed tomography; BUN, blood urea nitrogen; MRI, magnetic resonance imaging; BMI, body mass index; OR, odds ratio; CI, confidence interval. 


\section{Therapy of acute phase}

In this phase the choice of an appropriate care setting is mandatory. ${ }^{3,115}$ There are indications for admission or transfer in a setting of intensive/sub-intensive type: hypotension unresponsive to fluid loading, multi-organ failure, persistent SIRS, increased urea or creatinine and hematocrit, the presence of severe cardio-pulmonary comorbidities.

Referral to a specialist center is necessary if multiorgan failure persists and serious complications appear. It has been demonstrated that admission to a reference center is a factor that can influence the prognosis. ${ }^{116} \mathrm{The}$ cornerstones of treatment at this stage are: i) fluid resuscitation and support of the circle; ii) pain control; iii) nutrition; iv) infection control; v) monitoring and correction of hypoxia; vi) control of blood glucose.

\section{Fluid resuscitation}

Hydration is the only therapy that has been shown to improve survival in AP. ${ }^{3,117,118}$ Therefore, the first therapeutic approach consists of an accurate assessment of the state of hydration and hemodynamic balance, which can be compromised by vomiting, pain, diaphoresis, increased perspiration, vasodilation linked to the release of cytokines and pancreatic enzymes that alter the microcirculation and increase edema and extravascular fluid loss. ${ }^{3}$

The hydration therapy must be aggressive in the early stages (the first 6-12 h) to correct the blood volume and prevent the mesenteric hypoperfusion with the risk of ischemia and increased size of necrosis. ${ }^{119}$ In this way the hydration is also able to reduce the pain due to hypoperfusion.

Aggressive therapy consists in the infusion of 250$500 \mathrm{cc}$ of isotonic crystalloid hourly. Physiological saline or Ringer's lactate can be infused, but Ringer seems preferable unless in the presence of hypercalcemia. ${ }^{120}$ In cases of severe volume depletion a bolus of $20 \mathrm{~mL} / \mathrm{kg} / \mathrm{h}$ may be indicated for the first hour followed by a maintenance to $3 \mathrm{~mL} / \mathrm{kg} / \mathrm{h}$ (decrease to 1.5 $\mathrm{mL} / \mathrm{kg} / \mathrm{h}$ if BUN reduces) checking every 6-8 hours clinical status and vital signs. ${ }^{112,121}$

The assessment of the state of hydration and the need for replenishing liquids should be guided by clinical and can be facilitated by some laboratory parameters such as BUN, creatinine and hematocrit.

The following targets are considered indicators of adequate hydration: heart rate $<120 \mathrm{bpm}$, median arterial pressure between 65 and $85 \mathrm{mmHg}$, diuresis $>0.5-1 \mathrm{~mL} / \mathrm{kg} / \mathrm{h}$, decreased hematocrit and BUN. ${ }^{120,122}$ In a context of ICU type the variation of the stroke volume can be monitored for this purpose.

Infusion quantity and quality should consider degree of dehydration, electrolyte imbalances (including calcium and magnesium), diuresis and the presence of renal and/or cardiac complications and should be re- peatedly evaluated at regular intervals and adjusted in the first $24-48$ h. ${ }^{123}$

After the first $24 \mathrm{~h}$ aggressive rehydration showed no benefit and may be counterproductive because it can cause abdominal compartment syndrome, volume overload and pulmonary edema. ${ }^{117,121,124}$ If you cannot note a reduction in azotemia, you could be in the presence of acute tubular necrosis.

\section{Pain control}

Pain control is essential for the well being of the patient and to prevent further hemodynamic impairment. It can be achieved in an effective and safe way with opioids (meperidine, morphine and fentanyl). ${ }^{125}$

There are no studies that indicate the superiority of an opioid analgesic.

\section{Nutrition}

In the first 24-48 $\mathrm{h}$, in cases of mild to moderate pancreatitis, fluid supplementation may be useful. In these cases, the re-feeding can take place as soon as abdominal pain, nausea and vomiting stop and inflammation markers decrease. The diet should not necessarily be liquid or semi-liquid, but should be low-fat and low residue. ${ }^{126,127}$

In severe cases, artificial feeding is necessary with endoscopic or radiological positioning of naso-jejunal tube (preferred) and enteral nutrition formulas rich in protein and low in fat (with a caloric need of 25 $\mathrm{kcal} / \mathrm{kg}$ of ideal weight). This approach allows, with respect to the total parenteral nutrition (TPN), to maintain the integrity of the intestinal barrier, to reduce the risk of intestinal atrophy and bacterial/fungal translocation and to reduce the risk of sepsis related to the use of central venous access (CVC) and TPN. This way the risk of necrosis infection also reduces. ${ }^{128}$

In case it is impossible to position the naso-jejunal tube, data are in favor of nasogastric tube rather than the TPN: these data, however, should be confirmed. ${ }^{129}$

\section{Antibiotic therapy}

There are only two conditions in which antibiotic therapy is recommended: infection and prophylaxis of sterile necrosis. ${ }^{3,115,130,131}$

Infection of extrapancreatic sites (lung, urinary, biliary, CVC related) should be identified and treated with antibiotic therapy. Empirical therapy will be discontinued if culture tests are negative.

In case of pancreatic necrosis infection, antibiotic therapy can be empirical or targeted by means of CT guided fine-needle aspiration. CT-guided fine needle aspiration is not routinely recommended because combination of clinical and radiological data seems more accurate. $\mathrm{CT}$ guided fine-needle aspiration can be a reasonable approach in the case of no clinical improvement 
Table 2. Scoring system of acute pancreatitis gravity.

\section{Ranson's criteria}

A:

Age in years; white blood cell count; blood glucose; serum AST; serum ALT

$B$ :

Serum calcium; hematocrit fall; hypoxemia; BUN increased after IV fluid hydration; base deficit; sequestration of fluids If the score $\geq 3$, severe pancreatitis likely

If the score $<3$, severe pancreatitis is unlikely

\section{APACHE II score}

C:

1. $\mathrm{AaDO}_{2}$ or $\mathrm{PaO}_{2}$ (depending on $\mathrm{FiO}_{2}$ );

2. Temperature (rectal);

3. Mean arterial pressure;

4. $\mathrm{pH}$ arterial;

5. Heart rate;

6. Respiratory rate;

7. Sodium (serum);

8. Potassium (serum);

9. Creatinine

10. Hematocrit;

11. White blood cell count;

12. Glasgow Coma Scale

A score $<8$ is indicative of a mortality $<4 \%$

A score $>8$ of mortality between 11 and $18 \% 105$

\section{Systemic inflammatory response syndrome}

Features of SIRS are two or more of the following conditions:

- Temperature $>38.3^{\circ}$ or $<36^{\circ}$; heart rate $>90$ beats $/ \mathrm{min}$

- Respiratory rate of $>20$ breaths $/ \mathrm{min}$ or $\mathrm{PaCO}_{2}$ of $<32 \mathrm{mmHg}$;

- White blood cell count of $>12,000$ cells $/ \mathrm{mL},<4000$ cells $/ \mathrm{mL}$ or $>10$ percent immature form
It is one of the first scores used to determine the severity of the AP. The first five criteria (A) are assessed at the entrance and estimate the severity of the inflammatory process, while the following six criteria (B) are measured within the first $48 \mathrm{~h}$ and indicate the systemic effects of enzymes and toxins circulating. Although the Ranson's criteria continue to be used, a meta-analysis ${ }^{104}$ showed that this score is a poor predictor of gravity so as not to have advantages compared to clinical judgment

It was originally developed for critically ill patients admitted to intensive care. The point score is calculated from a patient's age and 12 routine physiological measurements $(\mathrm{C})$ : these were measured during the first $24 \mathrm{~h}$ after admission, information about previous health status, and some information obtained at admission (such as age). The calculation method is optimized for paper schemas, by using integer values and reducing the number of options so that data fits on a single-sheet paper form. Probably it is the largest system of severity score and the most studied in the AP. It has a good negative predictive value and a modest positive predictive value for predicting severe AP and can be run daily at the bedside. Decreasing values during the first $48 \mathrm{~h}$ suggest a slight AP, while increasing values suggest a serious AP

\section{BISAP score}

$D$ :

BUN $>25 \mathrm{mg} / \mathrm{dL}$; impaired mental status; $\geq 2$ SIRS criteria; age $>60$; pleural effusion present BISAP score of $0<1 \%$ risk of mortality

BISAP score $>=522 \%$ risk of mortality
The presence of SIRS is associated with an increased mortality in patients with AP. Initial studies have suggested that this score can reliably predict the severity of pancreatitis and have the advantage of being easily applied at the bedside every day. ${ }^{106}$ Compared to other more complicated score has the advantage of relying on clinical parameters that should always be considered in all patients admitted to internal medicine

BISAP score is an index of severity in acute pancreatitis score development of the bedside. ${ }^{107}$ A validation study of the BISAP score found that its performance was similar to APACHE II, Ranson's criteria, and the CT severity index system. ${ }^{108}$ A score of 1 is assigned to each of the following abnormalities in the patient (D)

\section{Harmless acute pancreatitis score}

E:

Rebound tenderness and/or guarding; normal hematocrit; normal serum creatinine

If all three are present it is possible to predict with 98\% accuracy a mild course ${ }^{109}$

Organ failure based score includes:

- Goris multiple organ failure score ${ }^{111}$

- Bernard score ${ }^{112}$

- SOFA score (sequential organ failure assessment) $)^{113}$

- Marshall organ dysfunction score ${ }^{114}$

The HAPS allows the emergency physician to identify patients who are low risk for the development of severe acute pancreatitis. This could have important implications when an emergency physician is considering allocating limited resources, such as intensive care unit beds. The HAPS considers the absence of $(\mathrm{E})$

The presence of early and persistent (for more than $48 \mathrm{~h}$ ) organ failure is widely regarded as a predictor of severe AP, increased mortality and prolonged hospitalization. ${ }^{110}$ There are several scoring systems that consider the presence of organ failure, ${ }^{111-114}$ all these consider the number and the degree of dysfunction of any body involved and also the use of certain inotropes or vasopressors, mechanical ventilation or dialysis

The modified score of Marshall which evaluates the degree of dysfunction of three organ systems (respiratory, cardiovascular and renal), is used in the recent revision of the classification criteria of Atlanta $^{4}$ to define the AP severe if $>2$. According to the authors this score has the advantage of being simple, universally applicable and allows an easy and objective stratification of patients but it has not been validated

CT severity index
The modified CT severity index is an extension of the original CT severity index that was developed by Balthazar and colleagues in 1994 for distinguishing mild, moderate and severe forms of acute pancreatitis. Scores are generated by estimating pancreatic inflammation and necrosis to give a score out of 10 . The finding of necrotizing pancreatitis on CT abdomen may modify the therapeutic approach to acute pancreatitis

AST, aspartate aminotransferase; ALT, alanine aminotransferase; BUN, blood urea nitrogen; SIRS, systemic inflammatory response syndrome; HAPS, harmless acute pancreatitis score; CT, computed tomography. 
after several weeks from onset in the absence of clear signs and symptoms of infected necrosis. ${ }^{132}$

The antibiotics of choice are those capable of penetrating necrosis (carbapenems, quinolones, metronidazole).

It is not recommended the routine administration of antifungal drugs as prophylaxis or therapy. ${ }^{133}$

\section{Other therapies}

Hypoxia should be corrected with supplements of oxygen and, if necessary, with mechanical ventilation, invasive and not. Hypoxia may be associated with acute respiratory distress syndrome (ARDS), pleural effusion, aggressive fluid resuscitation or superimposed pulmonary infections.

Hyperglycemia may be due to a pre-existing diabetes mellitus, pancreatic disease, stress or artificial nutrition and should be corrected with insulin therapy with target blood glucose of $140-180 \mathrm{mg} / \mathrm{dL}$.

The use of protease inhibitors, in the normal practice, has no clear role and there is no evidence to support it. ${ }^{134}$

\section{Treatment of complications}

The presence of local complications or organ failure sustained beyond 48-72 $\mathrm{h}$ requires the transfer in a reference center for the treatment of AP.

\section{Infectious complications}

The probability of developing an infected necrosis is independent of the degree of necrosis and is the main predictor of mortality.

Unlike previously believed, the infection may be an early complication of necrosis and Gram-negative intestinal bacteria are frequently involved. The use of antibiotics has expanded in the last decades. There are no indications of efficacy for probiotics.

If necessary, the timing of surgery must be carefully assessed in each case by a multidisciplinary team (gastroenterologists and surgeons).

In case of stable patients, necrosectomy should be deferred over the $4^{\text {th }}$ week, regardless of the location and extent of necrosis.

If the patient remains unstable despite antibiotic therapy, necrosectomy (percutaneous/endoscopic/laparoscopic or laparotomy surgery; if possible a minimally invasive approach is always preferred) is required. However, it would be better to postpone surgery as much as possible so that necrosis can be organized. ${ }^{132}$

\section{Abdominal compartment syndrome}

Abdominal compartment syndrome is defined as a persistent increase of intra-abdominal pressure $>20$ $\mathrm{mmHg}$ associated with the emergence of a new organ failure. It may be due to ascites, intestinal obstruction, peripancreatic inflammation and intensive hy- dration. The intra-abdominal pressure is measured in the bladder.

The treatment of compartment syndrome can be surgical or medical. ${ }^{135}$

Medical therapy includes viscera detention through naso-jejunal tube and rectal probe, prokinetic, endoscopic decompression, ultrafiltration or diuretics in case of fluid accumulation, analgesia and sedation until neuromuscular blockade to decrease the muscle tone of the abdominal wall.

Surgical therapy includes paracentesis, midline or subcostal laparostomy and subcutaneous fasciotomy. Surgical decompression can be lifesaving.

\section{Pancreatic duct disruption}

Pancreatic duct disruption produces pleural effusion, ascites and extension of the collections. Symptoms may include shortness of breath, abdominal pain, vomiting and dyspepsia.

A focal disconnection can be treated with stents placed endoscopically. If it occurs in the context of a large necrotic area, it requires a specialized surgical approach. ${ }^{136}$

\section{Pseudocyst}

Asymptomatic pseudocysts do not require therapy regardless of the location and size. The symptomatic pseudocyst is an indication for surgery.

\section{Thrombosis of splanchnic vessels}

The porto-spleno-mesenteric vein thrombosis occurs in $50 \%$ of patients with necrotizing pancreatitis and is rare in the absence of necrosis. Treatment consists in anticoagulants, which seem safe but ineffective. ${ }^{137}$

\section{Pseudoaneurysms}

Pseudoaneurysms are rare but they can lead to fatal consequences. They are responsible for severe gastrointestinal bleeding and sudden reductions in hematocrit. The treatment of choice is transcatheter arterial embolization. In case of failure the surgical approach is necessary.

\section{Systemic complications}

They are represented by the following clinical conditions: decompensated diabetes mellitus, exacerbation of preexisting comorbidities, extrapancreatic infections, alcohol withdrawal, respiratory failure due to ARDS, atelectasis or pleural effusion.

\section{Urgent surgical indication in acute pancreatitis}

Urgent surgical indications in acute pancreatitis are the following two: i) the biliary obstruction associated with cholangitis unresolved by ERCP; ii) infected 
necrosis with clinical deterioration associated with persistent signs of infection ( $>48-72 \mathrm{~h}$ ). Postponing surgery in conditions of stability (after at least four weeks) is demonstrated to be beneficial.

The conditions in which surgery may be necessary are: biliary obstruction with cholangitis; necrosectomy of infected necrosis; cholecystectomy in case of gallstone pancreatitis although already performed sphincterotomy (indicated at the admission); abdominal compartment syndrome unresponsive to conservative therapy; bleeding from pseudoaneurysms; intestinal ischemia; ductal disruption; fistula of the colon; symptomatic pseudocyst; ongoing gastric outlet, intestinal or biliary obstruction due to mass effect. It should be noted that in case of surgery for abdominal compartment syndrome, bleeding or intestinal ischemia, necrosectomy of sterile necrosis is not recommended, because it can cause necrosis infection.

\section{Therapy of predisposing factors}

\section{Gallstones}

In the case of choledocholithiasis with obstruction of the bile duct associated with pancreatitis and cholangitis, an urgent ERCP with sphincterotomy (within 24-72 h) is indicated. This indication is not valid if there are gallstones without cholangitis. ${ }^{138}$ ERCP should be considered, in a stable patient with clinical signs of obstruction. To reduce the risk of postERCP pancreatitis, pancreatic duct stents ${ }^{139}$ and nonsteroidal anti-inflammatory drugs (NSAIDs) given rectally have been found to be useful, especially in patients at high risk of severe pancreatitis. Probably NSAIDs should be administered before the procedure (indomethacin $100 \mathrm{mg}$ or diclofenac $100 \mathrm{mg}$ ). ${ }^{140}$

ERCP is also indicated in case of residual common bile duct stones after cholecystectomy. ERCP indications for acute biliary pancreatitis are: pancreatitis with biliary obstruction signs associated with fever (urgent-within 24-72 h); pancreatitis with biliary persistent signs of biliary obstruction (election- timing undefined).

In gallstone-associated pancreatitis, cholecystectomy should be performed (even in those who underwent ERCP) ${ }^{141}$ with a timing based on the pancreatitis severity: during the same hospitalization in case of mild pancreatitis; later for other grades of severity. Surgery should be performed after resolution of the acute inflammatory phase or after stabilization and organization of necrosis. It can be performed during the intervention of necrosectomy if indicated. ${ }^{142-144}$

Cholecystectomy may be performed after two episodes of acute idiopathic pancreatitis if there is an increase in transaminases and cholestasis markers (suspected microlithiasis).

Sphincterotomy, that reduces the risk of pancreatitis recurrence, may be adequate in patients with mul- tiple comorbidities or in the frail elderly, in which the operative risk is high. ${ }^{143}$

\section{Alcoholism}

It is advisable to prevent alcohol withdrawal syndrome and reintegration of thiamine.

\section{Hypertriglyceridemia}

The levels of plasma triglycerides $>1000 \mathrm{mg} / \mathrm{dL}$ should be considered as the cause for acute pancreatitis. In this case, apheresis, insulin and heparin (even combined) are suggested in addition to supportive therapy for pancreatitis early treatment with fibrates. ${ }^{144-146}$

\section{Hypercalcemia}

Causes of hypercalcemia should be identified and treated.

\section{The management of the patient with pancreatitis: rationale and objectives}

Acute pancreatitis is a dynamic clinical entity whose severity may vary during hospital stay. Its management may require the cooperation of many specialties (emergency medicine, internal medicine, gastroenterology, surgery, anesthesiologists) in order to ensure the right setting for the right patient, so that each possible complication may be treated in the best way. Therefore, a common language is of pivotal importance to clarify diagnosis, severity and complications and to exactly stratify the patients with the aim of guaranteeing a standard of care, under the therapeutic and diagnostic profile.

\section{The management of the patient with pancreatitis: methodology}

In order to provide evidence-based recommendations on the management of patients with acute pancreatitis, first of all we verified the existence of guidelines (GL) on this topic.

With this aim we performed a bibliographic search on this guidelines database: i) Scottish Intercollegiate Guidelines Network (SIGN); ii) Institute for Clinical Systematic Improvement (ICSI); iii) National Institute for Health and Clinical Excellence (NICE) (NHS evidence); iv) National Guideline Cleringhouse; v) Canadian Medical Association, CMA infobase; vi) New Zealand Guidelines Group; vii) National System Guidelines; viii) Clinical Practice Guidelines Portal; ix) eGuidelines.

The search has been independently performed by four authors using the following key words: pancreatitis or pancreas where the database included the pos- 
sibility of search, and manually examining the guidelines regarding the digestive system disease or biliary tract disease in the other cases. Given the poor result, we conducted further research using both Google search engine and the general database Medline, PubMed using the following search strategies: i) in the first case: pancreatitis AND guidelines; ii) in the second case: pancreatitis [MeSH Terms] and Guideline as limit and pancreatitis [MeSH Terms] and Consensus Development Conference as filter.

The results have been analyzed by each author and then discussed. Thus we (so) realized that most studies $^{147,148}$ analyzed acute pancreatitis guidelines with the AGREE method, ${ }^{139}$ considering 2008 as a time limit of bibliography. We therefore decided to analyze with the AGREE method (Appraisal of Guidelines, Research and Evaluation II) only the GL produced after 2008. The AGREE method evaluates the respect of 23 items, collected in 6 domains, that consider explication of the purpose, clarity, involvement of all stakeholders, applicability, editorial independence and two final considerations on global evaluation. Each author evaluates the respect of each item with a score going from 1 (total disagreement) to 7 (total agreement); scores given by each author are summed within each domain and related to maximum and minimum score that is possible to obtain in relation to the number of included item and the number of authors.

\section{The management of the patient with pancreatitis: results}

We identified 80 GL: 65 of these have been excluded because they did not regard management of AP. The remaining 15 are listed in Table 3 .

Finally, we decided to analyze the guidelines of the American College of Gastroenterology and International Association of Pancreatology/American Pancreatic Association (IAP/APA) using AGREE instrument. According to us, these guidelines are the most useful for clinical practice management of AP. They were evaluated using the AGREE instrument (Appraisal of Guidelines, Research and Evaluation II, 22) by 3 authors independently. Considering AGREE analyses of these two guidelines (Table 4), the domains 1 (scope and purpose) 4 (clarity of presentation) and 6 (editorial independence) revealed high score; on the contrary the domain 2 (stakeholder involvement) showed a low one, because involvement of target population points of view was not enough considered. Both guidelines are exhaustive in rigor of development (domain 3). American College of Gastroenterology (ACG) guidelines are inferior to IAP for GL assessment before publication by external experts and for procedure description of updating the GL. Regarding applicability (domains 5) both GL have some limitations about description of factors that may facilitate or impede the diffusion of GL and about explaining indicators for monitoring them. Overall, we found that IAP/APA guidelines fit best to our clinical setting (each author assigned 6 score $v s 5$ ).

The two works are discussed together for the presence of common shares. Greater emphasis will be as in the case of differences between the two guidelines on same subject (Table 5). A careful reading of the original articles is always advisable especially in the case of specific interest. Below we will list the main news emerged from the reading of the two guidelines regarding the clinical approach to the patients with acute pancreatitis.

\section{Clinical approach to patients with pancreatitis}

The management of pancreatitis consisted of four steps: i) diagnosis and evaluation etiology of pancreatitis; ii) initial evaluation and prognostic assessment; iii) initial management: fluid therapy, role of antibiotics and nutritional support; iv) management of complications.

\section{Diagnosis and evaluation etiology of pancreatitis (Grade 1B, strong agreement)}

The diagnosis of AP is possible when two of the following three criteria are present: i) abdominal pain consistent with the disease; ii) serum amylase and/or lipase greater than three times the upper limit of normal. Lipase is more specific. Possible modifications of the limit are admitted for diabetic patients who normally have a higher average value; iii) characteristic findings from abdominal imaging.

Concerning etiology, since the most frequent cause of AP is cholelithiasis and alcohol intake, an abdomen ultrasound examination should always be performed in the patient referred to the hospital for AP in addition to blood tests on cholestasis (ALT $>150 \mathrm{U} / \mathrm{L}$ in the first $48 \mathrm{~h}$ represents a positive predictive value $>85 \%$ ).

In the absence of clinical history of alcohol or gallstones, a careful medical history should be performed in order to identify previous unknown episodes of AP, use of drugs, abdominal trauma, infections, recent invasive procedures such as ERCP and other laboratory parameters, such as serum triglyceride (considered the etiology if $>1000 \mathrm{mg} / \mathrm{dL}$ ) and calcium.

The tumor etiology must always be regarded as possible cause of AP especially given the decreasing age average of onset of pancreatic adenocarcinoma or other malignant or benign tumors.

In case of idiopathic pancreatitis diagnosis there is no consensus about referring patients to a center of excellence. The guidelines IAP/APA recommend EUS that can point out micro lithiasis $<3 \mathrm{~mm}$, neoplasia or chronic pancreatitis and in case of negative examina- 
Table 3. Guidelines found on databases of guidelines, PubMed and Google.

\begin{aligned} \hline Databases of guidelines & ACR Appropriateness Criteria $囚$ acute \\ & pancreatitis; 1998 (revised 2013). \\ & NGC:010143 American College of Radiology \\ & - Medical Specialty Society \end{aligned}

The role of endoscopy in the evaluation of suspected choledocholithiasis; 2010 Jan.

NGC:008328 American Society for

Gastrointestinal Endoscopy - Medical

Specialty Society

PubMed/Google IAP/APA evidence-based guidelines for the management of acute pancreatitis

Pancreatology 2013;2013:e1-e15

Classification of acute pancreatitis-2012: revision of the Atlanta classification and definitions by international consensus. Gut 2013;62:102-11
It represents the 2013 review of the original criteria of 98 , and divides the clinical condition in four scenarios identifying what are the techniques of image to be taken for each scenario. The focus of the guidelines is on the diagnosis and subsequent assessment of pcs with AP. It is definitely useful because it adds information on diagnostic tools in different AP onset

Guideline for the practice of endoscopy, developed by the American Society for Gastrointestinal Endoscopy by using an evidence based methodology

The update of 2002 IAP/APA guidelines is based on an evidence-based approach to AP management. It includes the modified Atlanta criteria and the 38 recommendations regarding 12 aspects of AP. This guideline is graded with the GRADE method

It is the revision of the 1998 classification criteria of Atlanta. It is a consensus document made through an iterative process and web-based of a working group consisting of 11 national and international pancreatic society. The main goal is to provide unique classification criteria. It includes a clinical assessment of the severity of the AP proposing objective criteria (and therefore measurable and quantifiable) to describe the complications of local AP. It is not a guideline management of AP, neither gives therapeutic indications. It is included in two more recent guidelines, ACG and IAP/APA

SEMICYUC 2012. Recommendations for intensive care management of acute pancreatitis.

Med Intensiva 2013;37:163-179

It deals with the intensive approach to acute pancreatitis, including the multidisciplinary management of the patient, indications on early admission in ICU and therapy in critically ill patients. The section on the role of percutaneous drainage of necrosis is complete

Clinical pathways for acute pancreatitis. Recommendations for early multidisciplinary management.

Med intensiva 2012:36:351-357

It does not represent a true guideline. It is based on the recommendations address to 2005 guidelines SEMICYUC ICU. It was proposed by Petrov et al. in 2010 as classification criteria

Guidelines for specialized nutritional and It deals with nutrition in critically ill patients metabolic support in the critically-ill patient.

Update semicyuc-senpe consensus: severe acute pancreatitis

Nutr Hosp 2011;26:32-36

Practical guidelines for acute pancreatitis (official guidelines of the Italian association for the study of the pancreas regarding the medical, endoscopic and surgical management of acute pancreatitis). Pancreatology 2010;10:523-535

The document is not a real guideline but a review of the existing guidelines on the management of acute pancreatitis using ADAPTE method, in order to adapt them to Italian reality. The literature search was conducted looking all guidelines 1996-2007 in pub med cochrane library and other databases. The guidelines selected (9 from 21) were evaluated using the AGREE instrument and the working group concluded that all 9 guidelines met criteria to answer specific clinical questions about acute pancreatitis

ESPEN guidelines on parenteral nutrition pancreas clinical nutrition 2009;28:428-435

It concerns nutritional aspects in patient with acute pancreatitis

AGA institute medical position statement on acute pancreatitis.

Gastroenterology 2007;132:2019-2021

These recommendations are written in order to provide evidence based on prospective

Practice guidelines in acute pancreatitis. American journal of gastroenterology 2006

It considers various aspects of acute pancreatitis such as diagnoses, assessment of risk factors and severity, supportive care therapy, fluids therapy, the indication of ICU, nutrition, antibiotics, necrosis treatment

ESPEN guidelines on parenteral nutrition Pancreas Clinical Nutrition 2006;25:275-284

$J P N$ guidelines for the management of acute pancreatitis.

J Hepatobiliary Pancreat Surg 2006;13:56-60

UK guidelines for the management of acute It represents the revision of the previous 1998 guidelines, updated pancreatitis.

Gut 2005;54 suppl III
It concerns nutritional aspects in patient with acute pancreatitis

It contains several elements which are inconsistent with the latest guidelines, especially regarding therapeutic aspects to 2003

AP, acute pancreatitis; ACR, American College of Radiology; IAP/APA, International Association of Pancreatology/American Pancreatic Association; ACG, American College of Gastroenterology; ICU, Intensive Care Unit. 
tion, a secretin-stimulated-magnetic resonance cholangiopancreatography (MRCP) as a second step to identify rare morphologic abnormalities. If etiology remains unidentified, genetic counseling should be considered, especially after a second AP attack.

\section{Initial evaluation and prognostic assessment}

Hemodynamic status should be assessed immediately upon presentation and resuscitative measures begun as needed. Risk assessment should be performed to stratify patients into higher- and lower-risk categories to assist triage, such as admission to an intensive care setting (strong recommendation, moderate quality of evidence).

The best score to predict severe acute pancreatitis on admission and at 48 hours is the presence of SIRS. Persistent ( $>48 \mathrm{~h}$ ) SIRS is associated with multi-organ failure and mortality (25\%) in acute pancreatitis (Grade 2B, weak agreement).
The best strategy to predict outcome of acute pancreatitis during admission is a 3-dimension approach combining: i) host risk factors (e.g., age, co-morbidity, body mass index); ii) clinical risk stratification (e.g., persistent SIRS); iii) monitoring response to initial

Table 4. Score.

\begin{tabular}{lcc}
\hline Domain & IAP/APA & ACG \\
\hline 1 & $94 \%$ & $92 \%$ \\
\hline 2 & $58 \%$ & $47 \%$ \\
\hline 4 & $91 \%$ & $67 \%$ \\
\hline 5 & $97 \%$ & $100 \%$ \\
\hline 6 & $24 \%$ & 0 \\
\hline $\begin{array}{l}\text { IAP/APA, International Association of Pancreatology/American Pancreatic Associa- } \\
\text { tion; ACG, American College of Gastroenterology. }\end{array}$
\end{tabular}

IAP/APA, International Association of Pancreatology/American Pancreatic Association; ACG, American College of Gastroenterology.

Table 5. Difference between the two main guidelines.

\begin{tabular}{|c|c|c|}
\hline & Guidelines of ACG & Guidelines of IAP/APA \\
\hline If diagnosis of idiopathic pancreatitis & $\begin{array}{l}\text { Refer patient to centers of expertise; } \\
\text { Genetic testing may be considered in } \\
\text { young patients ( }<30 \text { years old) if no cause } \\
\text { is evident and a family history of } \\
\text { pancreatic disease is present (conditional } \\
\text { recommendation, low quality of evidence) }\end{array}$ & $\begin{array}{l}\text { If EUS is negative, secretin-stimulated-MRCP } \\
\text { can be used to identify rare morphologic } \\
\text { abnormalities } \\
\text { If etiology remains unidentified, genetic } \\
\text { counseling should be considered, especially after } \\
\text { a second attack of AP (Grade } 2 \mathrm{C} \text {, weak } \\
\text { agreement) }\end{array}$ \\
\hline Definition of specialist center & None & $\begin{array}{l}\text { High volume center with up-to-date intensive care } \\
\text { facilities including options for organ replacement } \\
\text { therapy, and with daily access to interventional } \\
\text { radiology, interventional endoscopy with EUS } \\
\text { and ERCP assistance as well as surgical expertise } \\
\text { in managing necrotizing pancreatitis (Grade } 2 \mathrm{C} \text {, } \\
\text { weak agreement) }\end{array}$ \\
\hline $\begin{array}{l}\text { Prevent severe post-ERCP pancreatitis } \\
\text { in high-risk patients }\end{array}$ & $\begin{array}{l}\text { Pancreatic duct stents and/or post-procedure } \\
\text { rectal non-steroidal anti-inflammatory drug } \\
\text { suppositories (conditional recommendation, } \\
\text { moderate quality of evidence) }\end{array}$ & None \\
\hline
\end{tabular}

The role of antibiotics in acute pancreatitis - Extrapancreatic infection, such as cholangitis, None catheter-acquired infections, bacteremia, urinary tract infections, pneumonia; (strong recommendation, high quality of evidence) - Infected necrosis (strong recommendation, low quality of evidence)

Consider and treat ACS None

ACS is defined as a sustained intra-abdominal pressure $>20 \mathrm{mmHg}$ (via the bladder with a maximal instillation volume of $25 \mathrm{~mL}$ of sterile saline) that is associated with new onset organ failure. It should be considered in mechanically ventilated patients with severe acute pancreatitis, especially in case of clinical deterioration. Medical treatment of ACS consists in decreasing intra-abdominal pressure

ACG, American College of Gastroenterology; IAP/APA, International Association of Pancreatology/American Pancreatic Association; EUS, endoscopic ultrasonography; MRCP, magnetic resonance cholangiopancreatography; AP, acute pancreatitis; ERCP, endoscopic retrograde cholangiopancreatography; NSAID, non-steroidal anti-inflammatory drug; ACS, abdominal compartment syndrome. 
therapy (e.g., persistent SIRS, blood urea nitrogen, creatinine) (Grade 2B, strong agreement).

Because of the absence of any available test to determine severity, assessing early fluid losses, hypovolemic shock, and organ dysfunction symptoms is crucial for any clinicians (Figure 2) to predict which patients with AP will develop severe disease.

Optimal timing for initial CT assessment is at least 72/96 h after onset of symptoms (Grade 1C, strong agreement).

Early CT may be useful to rule out bowel ischemia or intra-abdominal perforations in patients presenting with both acute pancreatitis and acute abdomen.

Indications for follow-up scanning (CT/MR) in acute pancreatitis are the following (Grade 1C, strong agreement): i) lack of clinical improvement; ii) clinical deterioration; iii) invasive intervention is considered, and only a portal venous phase (monophasic) is generally sufficient.

The optimal CT and MR protocol to detect necrosis should be applied.

Indications for ERCP and sphincterotomy are: patients with biliary pancreatitis and cholangitis (Grade $1 \mathrm{~B}$, strong agreement) and biliary pancreatitis with common bile duct obstruction (probably indicated) (Grade 1C, strong agreement).

The optimal timing of ERCP in patients with biliary pancreatitis without cholangitis is not known.

The urgent ERCP $(<24 \mathrm{~h})$ is required in patients with acute cholangitis (Grade $2 \mathrm{C}$, strong agreement).

As the exact timing of early ERCP (24/72 h) is not known, it is reasonable to await spontaneous improvement of biliary obstruction for 24/48 h. ERCP should be performed as soon as possible in patients with cholangitis.

The role of MRCP and EUS in biliary pancreatitis is to prevent some ERCPs that would otherwise be performed for suspected common bile duct stones in patients with biliary pancreatitis who do not have cholangitis, without influencing the clinical course. EUS is superior to MRCP in excluding the presence of small $(<5 \mathrm{~mm})$ gallstones. MRCP is less invasive, less operator-dependent and probably more widely available than EUS. Therefore, there is no clear superiority for either MRCP or EUS in clinical practice (Grade 2C, strong agreement).

\section{Initial management: fluid therapy, role of antibiotics and nutritional support}

Initial management includes patients' stabilization and their allocation according to AP severity.

\section{Fluid therapy}

Early fluid resuscitation is associated with decreased rates of persistent SIRS and organ failure (Grade 1C, strong agreement).
Ringer's lactate (Grade 1B, strong agreement) at the rate of $5-10 \mathrm{~mL} / \mathrm{kg} / \mathrm{h}(250-500 \mathrm{~mL} / \mathrm{h})($ Grade $1 \mathrm{~B}$, weak agreement) is the best fluid to use for initial fluid resuscitation in acute pancreatitis, unless cardiovascular and/or renal comorbidities exist. Early aggressive intravenous hydration is most beneficial during the first 12-24 h, and may have little benefit beyond. A more rapid repletion (bolus) may be necessary in a patient presented with hypotension and tachycardia for severe volume depletion. Fluid requirements should be reassessed at frequent intervals within 6 hours from admission and for the following 24-48 $\mathrm{h}$. The goal of aggressive hydration should be to decrease the blood urea nitrogen.

To assess the response to fluid therapy, one or more of the following points should be considered (Grade 2B, weak agreement): i) non-invasive measurement: heart rate $<120 / \mathrm{min}$; mean arterial pressure between 65 and $85 \mathrm{mmHg}(8.7-11.3 \mathrm{kPa})$; and urinary output $>0.5 / 1 \mathrm{~mL} / \mathrm{kg} / \mathrm{h}$; ii) invasive clinical targets: stroke volume variation and intrathoracic blood volume determination; iii) biochemical targets of hematocrit $35 / 44 \%$.

Indications for admission to an intensive care unit in acute pancreatitis are showed in Figure 3 (Grade $1 \mathrm{C}$, strong agreement).

\section{Antibiotics therapy}

Antibiotics should be given for an extrapancreatic infection, such as cholangitis, catheter-acquired infections, bacteremia, urinary tract infections, pneumonia. In patients with infected necrosis, antibiotics known to penetrate pancreatic necrosis, such as carbapenems, quinolones, and metronidazole, may be useful in delaying or avoiding intervention, thus decreasing morbidity and mortality.

Routine administration of antifungal agents along with prophylactic or therapeutic antibiotics is not recommended (conditional recommendation, low quality of evidence).

\section{Nutritional support}

In patients with predicted mild pancreatitis, oral feeding can be restarted once abdominal pain is resolved and inflammatory markers improved (Grade $2 \mathrm{~B}$, strong agreement). Feeding can be started with a full solid diet without waiting for normalization of lipase levels before restarting oral feeding.

In patients with predicted severe acute pancreatitis who require nutritional support enteral tube feeding should be the primary therapy and can be administered via either the nasojejunal or nasogastric route (Grade 1B, strong agreement).

If nasojejunal tube feeding is not tolerated and nutritional support is required, parenteral nutrition can be administered as second-line therapy (Grade 2C, 
strong agreement). Specific type of enteral nutrition or immunonutrition do not improve outcome in acute pancreatitis, either elemental or polymeric enteral nutrition formulations can be used in acute pancreatitis.

\section{Management of complications}

The indications for referral patients with AP to a specialist center (high volume center with up-to-date intensive care facilities including options for organ replacement therapy, (and with) daily access to interventional radiology, interventional endoscopy with EUS and ERCP assistance as well as surgical expertise in managing necrotizing pancreatitis) is severe acute pancreatitis and patients with local complication who may need interventional radiologic, endoscopic, or surgical intervention.
Indications for intervention in necrotizing pancreatitis (either radiological, endoscopic or surgical)

Common indications for intervention are (Grade $1 \mathrm{C}$, strong agreement): i) clinical suspicion or documented infected necrotizing pancreatitis with clinical deterioration, preferably when the necrosis has become walled-off; ii) presence of organ failure for several weeks after the onset of acute pancreatitis also in the absence of documented infected necrotizing pancreatitis.

Less common indications for intervention are (Grade 1C, strong agreement): i) abdominal compartment syndrome; ii) acute bleeding; iii) bowel ischemia; iv) gastric, intestinal, or biliary obstruction due to mass effect from large walled-off necrosis (arbitrarily $>4 / 8$ weeks after onset of pancreatitis).

\section{Patient characteristics}

Age $>55$ years
Obesity $\left(\mathrm{BMI}>30 \mathrm{~kg} / \mathrm{m}^{2}\right.$ )

Altered mental status

Comorbid disease

\section{The systemic inflammatory response syndrome}

Presence of $>2$ of the following criteria:

- pulse $>90$ beats $/ \mathrm{min}$

- respirations $>20 / \mathrm{min}$ or $\mathrm{PaCO}_{2}>32 \mathrm{mmHg}$

- temperature $>38^{\circ} \mathrm{C}$ or $<36^{\circ} \mathrm{C}$

- WBC count $>12,000$ or $<4000$ cells $/ \mathrm{mm}^{3}$ or $>10 \%$ immature

- neutrophils (bands)

\section{Laboratory findings}

BUN $>20 \mathrm{mg} / \mathrm{dL}$

\section{Rising BUN}

HCT $>44 \%$

\section{Rising HCT}

\section{Elevated creatinine}

\section{Radiology findings}

\section{Pleural effusions}

\section{Pulmonary infiltrates}

\section{Multiple or extensive extrapancreatic collections}

Figure 2. Clinical findings associated with a severe course for initial risk assessment (the presence of organ failure and/or pancreatic necrosis, defines severe acute pancreatitis). BMI, body mass index; WBC, white blood cell; BUN, blood urea nitrogen; HCT, hematocrit. 
Routine percutaneous needle aspiration of peripancreatic collections (to document infected necrotizing) is not indicated because clinical signs (i.e., persistent fever, increasing inflammatory markers) and imaging signs (i.e., gas in peripancreatic collections) are accurate predictors of infected necrosis in the majority of patients (Grade 1C, strong agreement).

\section{Indications for intervention (either radiological,} endoscopic or surgical) in sterile necrotizing pancreatitis

Indications for intervention (either radiological, endoscopic or surgical) in sterile necrotizing pancreatitis are (Grade 1C, strong agreement): i) gastric outlet, intestinal, or biliary obstruction due to mass effect of organized necrosis (i.e., arbitrarily $>4-8$ weeks after onset of acute pancreatitis); ii) persistent symptoms (e.g., pain, persistent malaise) in patients with organized necrosis without signs of infection (i.e., arbitrarily $>8$ weeks after onset of acute pancreatitis); iii) disconnected duct syndrome (i.e., full transection of the pancreatic duct in the presence of pancreatic necrosis) with persisting symptomatic (e.g., pain, obstruction) collection(s) with necrosis without signs of infections (i.e., arbitrarily $>8$ weeks after onset of acute pancreatitis).

The presence of asymptomatic pseudocysts and pancreatic and/or extrapancreatic necrosis does not warrant intervention, regardless of size, location, and/or extension.

\section{Timing of cholecystectomy}

After mild biliary pancreatitis cholecystectomy ap- pears safe and is recommended during index admission. Interval cholecystectomy after mild biliary pancreatitis is associated with a substantial risk of readmission for recurrent biliary events, especially recurrent biliary pancreatitis (Grade 1C, strong agreement).

After severe biliary pancreatitis with peripancreatic collections, cholecystectomy should be delayed until the collections resolve. In case they persist beyond 6 weeks, cholecystectomy can be performed safely (Grade 2C, strong agreement).

Cholecystectomy is also indicated in patients with biliary pancreatitis and previous history of sphincterotomy, since neither ERCP nor sphincterotomy prevent biliary colic and cholecystitis (Grade 2B, strong agreement).

\section{Conclusions}

The two guidelines considered above emphasize the latest clinical evidence in the context of diagnosis, etiology, and therapeutic approach to the patient with AP.

Their diffusion and application in the clinical setting can uniform clinical practice and improve patient outcomes.

In addition, the new definitions of severity and complications of AP used in these guidelines give us a universal language to properly stratify the patient for correct allocation.

For this reason, it is important that the internist knows diagnosis, management and treatment of this complex and multidisciplinary disease.

\section{One or more of the following parameters (106)}

1) Pulse $<40$ or $>150$ beats $/ \mathrm{min}$;

2) Systolic arterial pressure $<80 \mathrm{mmHg}(<10.7 \mathrm{kPa})$ or mean arterial pressure $<60 \mathrm{mmHg}(<8.0 \mathrm{kPa})$ or diastolic arterial pressure $>120 \mathrm{mmHg}(>16 \mathrm{kPa})$

3) Respiratory rate $>35$ breaths $/ \mathrm{min}$

4) Serum sodium $<110 \mathrm{mmol} / \mathrm{L}$ or $>170 \mathrm{mmol} / \mathrm{L}$

5) Serum potassium $<2.0 \mathrm{mmol} / \mathrm{L}$ or $>7.0 \mathrm{mmol} / \mathrm{L}$

6) $\mathrm{PaO}_{2}<50 \mathrm{mmHg}(<6.7 \mathrm{kPa})$

7) $\mathrm{pH}<7.1$ or $>7.7$

8) Serum glucose $>800 \mathrm{mg} / \mathrm{dL}$ ( $>44.4 \mathrm{mmol} / \mathrm{L}$ )

9) Serum calcium $>15 \mathrm{mg} / \mathrm{dL}(>3.75 \mathrm{mmol} / \mathrm{L})$

10) Anuria

11) Coma

Figure 3. Criteria for admission to intensive care unit. 


\section{References}

1. Vlada AC, Schmit B, Perry A, et al. Failure to follow evidence-based best practice guidelines in the treatment of severe acute pancreatitis. HPB (Oxford) 2013;15:822-7.

2. Stevens T, Parsi MA, Walsh RM. Acute pancreatitis: problems in adherence to guidelines. Cleveland Clinic J Med 2009;76 :697-704.

3. Tenner S, Baillie J, De Witt J. American College of Gastroenterology Guideline: management of acute pancreatitis. Am J Gastroenterol 2013;108:1400-15.

4. Banks PA, Bollen TL, Dervenis C. Acute Pancreatitis Classification Working Group Classification of acute pancreatitis 2012: revision of the Atlanta classification and definitions by international consensus. Gut 2013;62: 102-11.

5. Sekimoto M, Takada T, Kawarada Y. JPN guidelines for the management of acute pancreatitis epidemiology, aetiology, natural history and outcome predictors in acute pancreatitis. J Hepatobiliary Pancreat Surg 2006;13:10-24.

6. Horn GH, Hoem AD. Acute pancreatitis in Bergen, Norway: a study on incidence, etiology and severity. Scand J Surg 2004;93:29-33.

7. Lindkvist B, Appelros S, Manjer J. Trends in incidence of acute pancreatitis in a swedish population: is there really an increase? J Clin Gastroenterol Hepatol 2004;2: 831-7.

8. Yadav D, Lowenfels A. Trends in the epidemiology of the first attack of acute pancreatitis. A systematic review. Pancreas 2006;33:323-30.

9. Working group IAP/APA Acute pancreatitis guidelines. IAP/APA evidence based guidelines for the management of acute pancreatitis. Pancreatology 2013;13:e1-e15.

10. Yadav D, Lowenfles AB. The epidemiology of pancreatitis and pancreatic cancer. Gastroenterology 2013;144: 1252-61.

11. Hong S, Qiwen B, Ying J. Body mass index and the risk and prognosis of acute pancreatitis: a meta-analysis. Eur J Gastroenterol Hepatol 2011;23:1136-43.

12. Whitcomb DC, Gorry MC, Preston RA. Hereditary pancreatitis is caused by a mutation in the cationic trypsinogen gene. Nat Genet 1996;14:141-5.

13. Bathia M, Wong FL, Cao Y. Pathophisiology of acute pancreatitis. Pancreatology 2005;5:132-44.

14. Perides G, Laukkarinen JM, Vassileva G. Biliary acute pancreatitis in mice is mediated by the G protein coupled cell surface bile acid receptor GPBAR1. Gastroenterology 2010;138:715.

15. Clemens DL, Wells MA, Sneider KJ. Molecular mechanisms of alcohol associated pancreatitis. World J Gastroint Pathophysiol 2014;5:147-57.

16. Andriulli A, Loperfido S, Napolitano G. Incidence rate of post ERCP complications: a systematic survey of prospective studies. Am J Gastroenterol 2007;102:1781-8.

17. Wong L, Tsai H. Prevention of post ERCP pancreatitis. World J Gastrointestinal Pathophysiol 2014;5:1-10.

18. Chaudhari S, Park J, Anand BS. Acute pancreatitis associated with interferon and ribavirin therapy in patients with chronic hepatits C. Dig Dis Sci 2004;49:1000-6.

19. Ando K, Kim SR, Imoto S. acute pancreatitis associated with peylated interferon and ribavirin treatment for chronic hepatitis $\mathrm{C}$, genotype $1 \mathrm{~b}$ with high viral load. Case Rep Gastroenterol 2009;21:372-6.

20. Vignon KK, Seddik H, Rouibaa F. Acute pancreatitis during pegylated interferon therapy in a patient with chronic hepatitis B. J Gastointestinal Liver Dis 2009; 18:512.

21. Chou JW, Cheng KS, Huang CW. Sorafenib-induced Acute pancreatitis: a case report and review of the literature. Intern Med 2016;55:623-7.

22. Sevin A, Chen A, Atkinson B. Tyrosine kinase inhibitor induced pancreatitis J Oncol Pharm Pract 2013;19:257-60.

23. Ravi Kanth VV, Reddy Nageshwar D. Genetics of acute and chronic pancreatitis: an update. World J Gastrointest Pathophysiol 2014;5:427-37.

24. Jain P, Nijhawan S, Rai RR, et al. Acute pancreatitis in acute viral hepatitis. World J Gastroenterol 2007;13: 5741-4.

25. Mishra A, Saigal S, Gupta R, et al. Acute pancreatitis associated with viral hepatitis: a report of six cases with review of literature. Am J Gastroenterol 1999;94:2292-5.

26. Shimoda T, Shikata T, Karasawa T, et al. Light microscopic localization of hepatitis $B$ virus antigens in the human pancreas. Possibility of multiplication of hepatitis B virus in the human pancreas. Gastroenterology 1981;81:998-1005.

27. Yuen M-F, Chan T-M, Hui C-K, et al. Acute pancreatitis complicating acute exacerbation of chronic hepatitis B infection carries a poor prognosis. J Viral Hepat 2001;8: 459-64.

28. Swaroop VS, Chari ST, Clain JE. Severe acute pancreatitis. JAMA 2004;291:2865.

29. Banks PA. Acute pancreatitis. In: Lankisch PG, Banks PA, eds. Diagnosis in pancreatitis. New York: SpringerVerlag; 1998. p 75.

30. Banks PA, Freeman ML. Practice parameters committee of the American College of Gastroenterology. Practice guidelines in acute pancreatitis. Am J Gastroenterol 2006;101:2379.

31. Lankisch PG, Schirren CA, Kunze E. Undetected fatal acute pancreatitis: why is the disease so frequently overlooked? Am J Gastroenterol 1991;86:322.

32. Lankisch PG, Müller CH, Niederstadt H, Brand A. Painless acute pancreatitis subsequent to anticholinesterase insecticide (parathion) intoxication. Am J Gastroenterol 1990;85:872.

33. Kesavan CR, Pitchumoni CS, Marino WD. Acute painless pancreatitis as a rare complication in Legionnaires disease. Am J Gastroenterol 1993;88:468.

34. Mookadam F, Cikes M. Images in clinical medicine. Cullen's and Turner's signs. N Engl J Med 2005;353: 1386.

35. Dickson AP, Imrie CW. The incidence and prognosis of body wall ecchymosis in acute pancreatitis. Surg Gynecol Obstet 1984;159:343.

36. Clavien PA, Robert J, Meyer P, et al. Acute pancreatitis and normoamylasemia. Not an uncommon combination. Ann Surg 1989;210:614-20.

37. Winslet M, Hall C, London NJM. Relation of diagnostic serum amylase levels to aetiology and severity of acute pancreatitis. Gut 1992;33:982-6. 
38. Dervenis C, Johnson CD, Bassi C, et al. Diagnosis, objective assessment of severity, and management of acute pancreatitis. Santorini consensus conference. Int J Pancreatol 1999;25:195.

39. Tenner S, Fernandez-del Castillo C, Warshaw A, et al. Urinary trypsinogen activation peptide (TAP) predicts severity in patients with acute pancreatitis. Int J Pancreatol 1997;21:105.

40. Huang QL, Qian ZX, Li H. A comparative study of the urinary trypsinogen-2, trypsinogen activation peptide, and the computed tomography severity index as early predictors of the severity of acute pancreatitis. Hepatogastroenterology 2010;57:1295.

41. Khan Z, Vlodov J, Horovitz J, et al. Urinary trypsinogen activation peptide is more accurate than hematocrit in determining severity in patients with acute pancreatitis: a prospective study. Am J Gastroenterol 2002;97:1973.

42. Kylänpää-Bäck M, Kemppainen E, Puolakkainen P, et al. Reliable screening for acute pancreatitis with rapid urine trypsinogen-2 test strip. Br J Surg 2000; 87:49.

43. Mayumi T, Inui K, Maetani I, et al. Validity of the urinary trypsinogen-2 test in the diagnosis of acute pancreatitis. Pancreas 2012;41:869.

44. Kemppainen E, Hedström J, Puolakkainen P, et al. Increased serum trypsinogen 2 and trypsin 2-alpha 1 antitrypsin complex values identify endoscopic retrograde cholangiopancreatography induced pancreatitis with high accuracy. Gut 1997;41:690.

45. Kemppainen E, Hedström J, Puolakkainen P, et al. Urinary trypsinogen-2 test strip in detecting ERCP-induced pancreatitis. Endoscopy 1997;29:247.

46. Toouli J, Brooke-Smith M, Bassi C, et al. Guidelines for the management of acute pancreatitis. J Gastroenterol Hepatol 2002;17:S15.

47. Stevens T, Parsi MA, Walsh RM. Acute pancreatitis: problems in adherence to guidelines. Cleve Clin J Med. Dec 2009; 76:697-704.

48. Nuernberg D, Ignee A, Dietrich CF. Ultrasound in gastroenterology. Biliopancreatic system. Med Klin (Munich) 2007;102:112-26.

49. Neoptolemos JP, Hall AW, Finlay DF, et al. The urgent diagnosis of gallstones in acute pancreatitis: a prospective study of three methods. Br J Surg 1984;71:230-3.

50. Martínez-Noguera A, Montserrat E, Torrubia S, et al. U1trasound of the pancreas: update and controversies. Eur Radiol 2001;11:1594-606.

51. Bruno C, Minniti S, Schenal G. The role of ultrasound in acute pancreatitis. Med Radiol 2009;33-47.

52. Scuderi M, Sofia M, Latteri S, et al. Pancreatite acuta. Manuale Italiano di Ecografia Internistica, II ed. Sassari: Delfino ed.; 2013. pp 250-258.

53. Balthazar EJ. CT diagnosis of and staging of acute pancreatitis. Radiol Clin North Am 1989;27:19-37.

54. Bertolotto M, D’Onofrio M, Martone E, et al. Ultrasonography of the pancreas. Doppler imaging. Abdom Imaging 2007;32:161-70.

55. Ros E, Navarro S, Bru C, et al. Occult microlithiasis in "idiopathic" acute pancreatitis: Prevention of relapses bycholecystectomy or ursodeoxycholic acid therapy. Gastroenterology 1991;101:1701.
56. Rickes S, Wermke W. Differentiation of cystic pancreatic neoplasms and pseudocysts by conventional and echoenhanced ultrasound. J Gastroenterol Hepatol 2004;19: 761-6.

57. Tana C, D'Alessando P, Tartaro A, et al. Sonographic assessment of a suspected biloma: A case report and review of the literature. World J Radiol 2013;5:220-5.

58. Cai D-M, Parajuly SS, Ling W-W, et al. Diagnostic value of contrast enhanced ultrasound for splenic artery complications following acute pancreatitis. World J Gastroenterol 2014;20:4.

59. D’Onofrio M, Zamboni G, Faccioli N, et al. Ultrasonography of the pancreas. 4. Contrast-enhanced imaging. Abdom Imaging 2007;32:171-81.

60. D’Onofrio M, Zamboni G, Tognolini A, et al. Massforming pancreatitis: value of contrast-enhanced ultrasonography. World J Gastroenterol 2006;12:4181-4.

61. Rickes S, Uhle C, Kahl S, et al. Echo enhanced ultrasound: a new valid initial imaging approach for severe acute pancreatitis Gut 2006;55:74-8.

62. Seicean A, Badea R, Stan-Iuga R, et al. The added value of real-time harmonics contrast-enhanced endoscopic ultrasonography for the characterization of pancreatic diseases in routine practice. J Gastrointestin Liver Dis 2010;19:99-104.

63. Piscaglia F. The EFSUMB Guidelines and Recommendations on the Clinical Practice of Contrast Enhanced Ultrasound (CEUS): update 2011 on non-hepatic applications. Ultrashall Med 2012;33:33-59.

64. D’Onofrio M, Gallotti A, Pozzi Mucelli R. Imaging techniques in pancreatic tumors. Expert Rev Med Devices 2010;7:257-73.

65. Hocke M, Ignee A, Dietrich CF. Contrast-enhanced endoscopic ultrasound in the diagnosis of autoimmune pancreatitis. Endoscopy 2011;43:163-5.

66. Ripollés T, Martínez MJ, López E, et al. Contrast-enhanced ultrasound in the staging of acute pancreatitis. Eur Radiol 2010;20:2518-23.

67. Fusaroli P, Vallar R, Togliuani T, et al. Scientific publications in endoscopic ultrasonography: a 20-year global survey of the literatute. Endoscopy 2002;34:451-6.

68. Garrow D, Miller S, Sinha D, et al. Endoscopic ultrasound: a metanalysis of test performance in suspected biliary obstruction. Clin Gastroenterol Hepatol 2007;5:616-23.

69. Cennamo V, Testoni PA. Gestione Endoscopica della calcolosi della via biliare. Giornal Italian End Dig 2012; 35:71-5.

70. Tse F, Liu L, Barkun AN, et al. EUS: a meta-analysis of test performance in suspected choledocholithiasis. Gastrointes Endosc 2008;67:235-44.

71. Harewood GC, Wiersema MJ. A cost analysis of endoscopic ultrasound in the evaluation of pancreatic head adenocarcinoma. Am J Gastroenter 2001;96:2651-6.

72. Quirk D, Rattner D, Fernandez C, et al. The use of endocopic ultrasonography to reduce the cost of treating ampullary tumors. Gastrointest Endosc 1996;43:429.

73. Harewood GC, Wiersema MJ. Endosonography-guided fine needle aspiration biopsy in the evaluation of pancreatic masses. Am J Gastroenterol 2002;97:1386-91. 
74. Tio TL, Reeders WA, Sie LH, et al. Endosonography in the clinical staging of Klatskin tumor. Endoscopy 1993; 25:81-5.

75. Tamada K, Ido K, Ichiyama M, et al. Assessment of portal vein invasion by bile duct cancer using intraductal ultrasonography. Endoscopy 1995;27:573-8.

76. Legmann P, Vignaux O, Dousset B, et al. Pancreatic tumors: comparison of dual-phase helical CT and endoscopic sonography. AJR Am J Roentgenol 1998;170: 1315-22.

77. Brugge WR, Lee MJ, Kelsey PB, et al. The use of EUS to diagnose malignant portal venous system invasion by pancreatic cancer. Gastrointest Endoscop 1996;43: 561-7.

78. Buscarini E, De Angelis C, Arcidiacono PG, et al. Multicenter retrospective study on endoscopic ultrasound complications. Dig Liv Dis 2006;38:762-7.

79. Seifert H, Dietrich C, Schmitt T, et al. Endoscopic ultrasound-guided one-step transmural drainage of cystic abdominal lesions with a large-channel echo endoscope. Endoscopy 2000;32:255-9.

80. Windsor JA. Search for prognostic markers for acute pancreatitis. Lancet 2000;355:1924.

81. Chauhan S, Forsmark CE. The difficulty in predicting outcome in acute pancreatitis. Am J Gastroenterol 2010;105:443.

82. Beger HG, Warshaw AL, Buchler MW, et al. Assessment of clinical severity and prognosis. In: Beger HG, Warshaw AL, Buchler MW, et al, eds. The pancreas. Oxford: Blackwell Science; 1998. p 489.

83. Papachristou GI, Papachristou DJ, Morinville VD, et al. Chronic alcohol consumption is a major risk factor for pancreatic necrosis in acute pancreatitis. Am J Gastroenterol 2006;101:2605.

84. Martínez J, Johnson CD, Sánchez-Payá J, et al. Obesity is a definitive risk factor of severity and mortality in acute pancreatitis: an updated meta-analysis Pancreatology. 2006;6:206.

85. Lankisch PG, Blum T, Maisonneuve P, Lowenfels AB. Severe acute pancreatitis: when to be concerned? Pancreatology 2003;3:102.

86. Isenmann R, Rau B, Beger HG. Early severe acute pancreatitis: characteristics of a new subgroup. Pancreas 2001;22:274.

87. Brown A, Orav J, Banks PA. Hemoconcentration is an early marker for organ failure and necrotizing pancreatitis. Pancreas 2000;20:367.

88. Remes-Troche JM, Duarte-Rojo A, Morales G, RoblesDíaz G. Hemoconcentration is a poor predictor of severity in acute pancreatitis. World J Gastroenterol 2005;11:7018.

89. Wu BU, Johannes RS, Sun X, et al. Early changes in blood urea nitrogen predict mortality in acute pancreatitis. Gastroenterology 2009;137:129.

90. Wu BU, Bakker OJ, Papachristou GI, et al. Blood urea nitrogen in the early assessment of acute pancreatitis: an international validation study. Arch Intern Med 2011; 171:669.

91. Muddana V, Whitcomb DC, Khalid A, et al. Elevated serum creatinine as a marker of pancreatic necrosis in acute pancreatitis. Am J Gastroenterol 2009;104:164.
92. Lankisch PG, Weber-Dany B, Maisonneuve P, Lowenfels AB. High serum creatinine in acute pancreatitis: a marker for pancreatic necrosis? Am J Gastroenterol 2010;105:1196.

93. Forsmark CE, Baillie J. AGA Institute technical review on acute pancreatitis. AGA Institute Clinical Practice and Economics Committee, AGA Institute Governing Board Gastroenterology 2007;132:2022.

94. Papachristou GI, Whitcomb DC. Predictors of severity and necrosis in acute pancreatitis. Gastroenterol Clin North Am 2004;33:871.

95. Talamini G, Uomo G, Pezzilli R, et al. Serum creatinine and chest radiographs in the early assessment of acute pancreatitis. Am J Surg 1999;177:7.

96. Bollen TL, Singh VK, Maurer R, et al. A comparative evaluation of radiologic and clinical scoring systems in the early prediction of severity in acute pancreatitis. Am J Gastroenterol 2012;107:612.

97. Tsuji Y, Yamamoto H, Yazumi S, et al. Perfusion computerized tomography can predict pancreatic necrosis in early stages of severe acute pancreatitis. Clin Gastroenterol Hepatol 2007; 5:1484.

98. Arvanitakis M, Koustiani G, Gantzarou A, et al. Staging of severity and prognosis of acute pancreatitis by computed tomography and magnetic resonance imaging-a comparative study. Dig Liver Dis 2007;39:473-82.

99. Arvanitakis M, Delhaye M, De Maertelaere V, et al. Computed tomography and magnetic resonance imaging in the assessment of acute pancreatitis. Gastroenterology 2004;126:715.

100. Manikkavasakar S, AlObaidy M. Magnetic resonance imaging of pancreatitis: an update Dig Liver Dis 2007;39:473-82.

101. Mounzer R, Langmead CJ, Wu BU, et al. Comparison of existing clinical scoring systems to predict persistent organ failure in patients with acute pancreatitis. Gastroenterology 2012;142:1476-82; quiz e15-6.

102. Wang X, Xu Y, Qiao Y, et al. An evidence-based proposal for predicting organ failure in severe acute pancreatitis. Pancreas 2013;42:1255-61.

103. De Bernardinis M, Violi V, Roncoroni L, et al. Discriminant power and information content of Ranson's prognostic signs in acute pancreatitis: a meta-analytic study. A Crit Care Med 1999;27:2272.

104. Banks PA, Freeman ML. Practice guidelines in acute pancreatitis. Practice Parameters Committee of the American College of Gastroenterology. Am J Gastroenterol 2006;101:2379.

105. Buter A, Imrie CW, Carter CR, et al. Dynamic nature of early organ dysfunction determines outcome in acute pancreatitis. Br J Surg 2002;89:298.

106. Wu BU, Johannes RS, Sun X, et al. The early prediction of mortality in acute pancreatitis: a large populationbased study. Gut 2008;57:1698.

107. Papachristou GI, Muddana V, Yadav D, et al. Comparison of BISAP, Ranson's, APACHE-II, and CTSI scores in predicting organ failure, complications, and mortality in acute pancreatitis. Am J Gastroenterol 2010;105:435.

108. Lankisch PG, Weber-Dany B, Hebel K, et al. The harm- 
less acute pancreatitis score: a clinical algorithm for rapid initial stratification of non severe disease. Clin Gastroenterol Hepatol 2009;7:702.

109. Johnson CD, Abu-Hilal M. Persistent organ failure during the first week as a marker of fatal outcome in acute pancreatitis. Gut 2004;53:1340.

110. Goris RJ, te Boekhorst TP, Nuytinck JK, Gimbrère JS. Multiple-organ failure. Generalized autodestructive inflammation? Arch Surg 1985;120:1109.

111. Bernard GR, Doig G, Hudson L, et al. Quantification of organ failure for clinical trials and clinical practice. Am J Respir Crit Care Med 1995; 151:A323.

112. Vincent JL, Moreno R, Takala J, et al. The SOFA (Sepsis-related Organ Failure Assessment) score to describe organ dysfunction/failure. On behalf of the Working Group on Sepsis-Related Problems of the European Society of Intensive Care Medicine. Intensive Care Med 1996;22:707.

113. Marshall JC, Cook DJ, Christou NV, et al. Multiple organ dysfunction score: a reliable descriptor of a complex clinical outcome. Crit Care Med 1995;23:1638.

114. Working group IAP/APA Acute pancreatitis guidelines. IAP/APA evidence based guidelines for the management of acute pancreatitis. Pancreatology 2013;13:e1-e15.

115. Singla A, Simons J, Li Y, et al. Admission volume determines outcome for patients with acute pancreatitis. Gastroenterology 2009;137:1995-2001.

116. Nasr JY, Papachristou GI. Early fluid resuscitation in acute pancreatitis: a lot more than just fluids. Clin Gastroenterol Hepatol 2011;9:633-4.

117. Wall I, Badalov N, Baradian R, et al Decreased mortality in acute pancreatitis related to early aggressive hidratation. Pancreas 2011;40:547-50.

118. Takeda K, Mikami Y, Fukuyama S, et al. Pancreatic ischemia associated with vasospasm in the early phase of human acute necrotizing pancreatitis. Pancreas 2005;30;40-9.

119. Wu BU, Hwang JQ, Gardner TH. Lactated Ringer's solution reduces systemic inflammation compared with saline in patient with acute pancreatitis. Clin Gastroenterol Hepatol 2011;9:710-7.

120. Mao EQ, Fei J, Peng YB, et al. Rapid hemodiluition is associated with increased sepsis and mortality among patients with severe acute pancreatitis. Chin Med J 2010;123:1639-44.

121. Papachristou GI, Muddana V, Yadav D, et al. Increased serum creatinine is associated with pancreatic necrosis in acute pancreatitis. Am J Gastroenterol 2010;105: 1451-2.

122. Eckerwall G, Olin H, Andersson B, et al. Fluid resuscitation and nutritional support during severe acute pancreatitis in the past: what have we learned and how can we do better? Clin Nutr 2006;25:497-504.

123. de Madaria E, Soler-Sala G, Sanchez Paya J, et al. Influence of fluid therapy on the prognosis of acute pancreatitis; a prospective cohort study. Am J Gastroenterol 2011;106:1843-50.

124. Basurto Ona X, Rigau Comas D, Urrútia G. Opioids for acute pancreatitis pain. Cochrane Database Syst Rev 2013;7: CD009179.
125. Jacobson BC, Vander Vliet MB, Hughes MD, et al. A prospective, randomized trial of clear liquids versus low-fat solid diet as the initial meal in mild acute pancreatitis. Clin Gastroenterol Hepatol 2007;5:946-51.

126. Eckerwall GE, Tingstedt BB, Bergenzaun PE, et al. Immediate oral feeding in patients with mild acute pancreatitis is safe and may accelerate recovery-a randomized clinical study. Clin Nutr 2007;26:758-63.

127. Al-Omran M, Albalawi ZH, Tashkandi MF, et al. Enteral versus parenteral nutrition for acute pancreatitis. Cochrane Database Syst Rev 2010;(1):CD002837.

128. Singh N, Sharma B, Sharma M, et al. Evaluation of early enteral feeding through nasogastric and nasojejunal tube in severe acute pancreatitis; a noninferiority randomized controlled trial. Pancreas 2012;41:153-9.

129. Wittau M, Mayer B, Scheele J, et al. Systematic review and meta-analysis of antibiotic prophilaxis in severe acute pancreatitis. Scand J Gastroenterol 2011;46:261-70.

130. Villatoro E, Mulla M, Larvin M. Antibiotic therapy for prophylaxis against infection of pancreatic necrosis in acute pancreatitis. Cochrane Database Syst Rev 2010;(5):CD002941.

131. Freeman MF, Werner J, van Santvoort HC, et al. Intervention for necrotizing pancreatitis. Summary of a multidisciplinary consensus conference. Pancreas 2012;8: 1176-94.

132. Trikudanathan G, Navaneethan U, Vege SS. Intra-abdominal fungal infections complicating acute pancreatitis: a review. Am J Gastroenterol 2011;106:1188.

133. Seta T, Noguchi Y, Shimada T, et al. Treatment of acute pancreatitis with protease inhibitors: a meta-analysis. Eur J Gastroenterol Hepatol 2004;16:1287.

134. Kirkpatrick AW, Roberts DJ, DE Wj, et al. Intra-abdominal hypertension and the abdominal compartment syndrome: updated consensus definitions and clinical practice guidelines from the World Society of the Abdominal Compartment Syndrome. Intensive Care Med $2013 ; 39: 1190-206$.

135. Varadarajulu S, Noone TC, Tutuian R, et al. Predictors of outcome in pancreatic duct disruption managed by endoscopic transpapillary stent placement. Gastrointest Endosc 2005;61:568-75.

136. Harris S, Nadkarni NA, Naina HV, Vege SS. Splanchnic vein thrombosis in acute pancreatitis: a single-center experience. Pancreas 2013;42:1251.

137. Tse F, Yuan Y. Early routine endoscopic retrograde cholangiopancreatography strategy versus early conservative management strategy in acute gallstone pancreatitis. Cochrane Database Syst Rev 2012;5:CD009779.

138. Choudary A, Bechtold ML, Arif M, et al. Pancreatic stents for prophylaxis against post-ERCP pancreatitis; a meta-analysis and systematic review. Gastroinst Endosc 2011;40:809-14.

139. Elmunzer BJ, Scheiman JM, Lehman GA, et al. A randomized trial of rectal indomethacin to prevent postERCP pnacreatitis. N Engl J Med 2012;366:1414-22.

140. Heider TR, Brown A, Grimm IS, Behrns KE. Endoscopic sphincterotomy permits interval laparoscopic cholecystectomy in patients with moderately severe gallstone pancreatitis. J Gastrointest Surg 2006;10:1-5. 
141. van Baal MC, Besselink MG, Bakker OJ, et al. Timing of cholecistystectomy after mild biliary pancreatitis: a systematic review. Ann Surg 2012; 255:860-6.

142. Bakker OJ, van Santvoort HC, Hagenaars JC, et al. Timing of cholecystectomy after mild biliary pancreatitis. Br J Surg 2011;98:1446-54.

143. Szczepiorkowski ZM, Winters JL, Bandarenko N, et al. Guidelines on the use of therapeutic apheresis in clinical practice-evidence-based approach from the Apheresis Applications Committee of the American Society for Apheresis. J Clin Apher 2010;25:83.

144. Mikhail N, Trivedi K, Page C, et al. Treatment of severe hypertriglyceridemia in nondiabetic patients with insulin. Am J Emerg Med 2005;23:415.
145. Jain D, Zimmerschied J. Heparin and insulin for hypertriglyceridemia-induced pancreatitis: case report. Sci World J 2009;9:1230.

146. Benjamin PT, Loveday MB, Srinivasa S, et al. High quantity and variable quality of guidelines for acute pancreatitis: a systematic review. Am J Gastroenterol 2010;105:1466-76.

147. Pezzilli R, Zerbi A, Di Carlo V, et al. Practical guidelines for acute pancreatitis. Pancreatology 2010;10:523-35.

148. Brouwers M, Kho ME, Browman GP, et al.; for the AGREE Next Steps Consortium. AGREE II: Advancing guideline development, reporting and evaluation in healthcare. Can Med Assoc J 2010. Available online July 5, 2010: http://www.agreetrust.org/agree-ii/ 